\title{
Dynamics of flares on late type dMe stars
}

\section{Constraints from spectrophotometry in the visible}

\author{
E. R. Houdebine \\ Department of Physics (Theoretical Physics), Oxford University, 1 Keble Road, Oxford OX1 3NP, UK
}

Received 12 November 2001 / Accepted 1 August 2002

\begin{abstract}
We investigate the spectral signatures of stellar flares in the wavelength range $3600 \AA$ to $4500 \AA$ and in broad band photometry. We study the phenomenology of the spectral signatures and we found that flares are best described by two main phases; an impulsive phase and a gradual phase, for which the physical properties are different. Important spectral differences between flares lead us to distinguish four main classes: (i) solar-like chromospheric or two-ribbon flares, (ii) white-light flares, (iii) combined white-light flares with distinct impulsive and gradual phases, and (iv) non solar-like flares (usually occuring on RS CVn type stars). We show how this classification corresponds to substantial differences in the physical properties of the flare components.

We compiled all available spectroscopic data for stellar flares. We found several new empirical correlations between the time lags in the spectral lines (rise and decay times). We found for instance that during the gradual phase, the rise time in the $\mathrm{H}_{\gamma}$ line is well correlated to the rise time in the $\mathrm{Ca}$ II $\mathrm{K}$ line, and that the $\mathrm{Ca}$ II $\mathrm{K}$ line is 1.63 times slower to rise than the $\mathrm{H}_{\gamma}$ line. Similar correlations were found between the rise and decay times in these lines. These correlations are evidence that there is a dominant mechanism commanding the temporal flux evolutions during the gradual phase of flares. This mechanism applies on time scales ranging from one minute to more than a hundred minutes.

We found correlations between the time lags and the maximum fluxes in the Johnson $U$-band and the spectral lines. These correlations show that the larger the flare the longer it takes to evolve.

We show that the maximum flux in the Johnson $U$-band correlates well, but not linearly with the maximum flux in the $\mathrm{H}_{\gamma}$ line during the impulsive phase, and over five orders of magnitude. We argue how this correlation can provide constraints on the currently available models. The spectral line maximum fluxes during the gradual phase also correlate with the Johnson $U$-band flux, which demonstrates that somehow the impulsive and gradual phases are physically linked. The correlation between the $\mathrm{H}_{\gamma}$ and CaII $\mathrm{K}$ line fluxes during the gradual phase and the lack of dependence of the flux ratio of those lines on flare magnitude reveal that larger flares are not "hotter" than smaller flares during the gradual phase.

We examine the behaviour of the spectral line widths: While the Ca II K line width shows essentially no detectable variation, the Balmer line widths show a complex dependency on the white light intensity. We distinguish three energy domains where the Balmer line widths exhibit a different behaviour: (i) for small flares, the widths remain rather constant as a function of white light intensity, which suggests that in this energy range the $U$-band is not a good diagnostic of the total energy release and that dense "kernels" do not dominate the Balmer emission, (ii) for medium size flares, the widths rapidly increase with the $U$-band flux up to about $15 \AA$, which indicates that emission in the Balmer lines is then dominated by kernel emission, and (iii) for large flares the widths decrease with increasing $U$-band flux, which suggests that the Balmer line emission is then increasingly dominated by a radiative pumping process between the white light and the Balmer lines.

Finally, we remark that the Balmer line fluxes are well correlated to the white light flux during the impulsive phase of flares, and not only at flare maximum. We obtain a power law correlation between the Balmer line and $U$-band fluxes that is evidence for either a common source ("kernels") or strongly related flare components.
\end{abstract}

Key words. stars: flare - stars: activity - stars: late-type

\section{Introduction}

Observations of stellar flares show evidence of great variety in their morphology. Some flares display a strong impulsive phase when white light is emitted (Rodonó et al. 1989; Houdebine 1992a), sometimes followed by a slower gradual phase when

^ e-mail: erh@thphys.ox.ac.uk emission lines are prominent (Hawley \& Pettersen 1991; Rodonó et al. 1989). Other flares are more like the solar tworibbon flares with no white light emission (Houdebine 1992a; Butler et al. 1986). More generally, stellar flares display a wide variety of spectral signatures and evolution time scales. Most often, intense white light emission is reported, impulsive in character, with a rise time from tens of seconds to a couple 
of minutes. On late type dwarfs, these white light flares share many common properties with their solar equivalent, and for that reason can be referred to as "solar type white-light flares". However, not all flares may be of solar type. On RS CVn systems, some more complex white light flares occur, lasting for a day or more and which are generally more powerfull (e.g. Foing et al. 1994).

In fact, the typical solar flare has no detectable signature in white light, but few such events have been detected on stars. Such flares were detected in spectroscopy (e.g. Houdebine 1992a; Butler et al. 1986), X-rays (e.g. Butler et al. 1988; Doyle et al. 1988b) or other spectral domains. Ironically, this latter class of flares is the least known for stars, conversely to the Sun, because of an observational bias, i.e., very little time has been dedicated to spectroscopic or X-ray observations in comparison to photometric observations. However, we must emphasize that there is also a strong contrast effect (Houdebine 1992a), in other words, many flares have been detected as white-light flares on dMe stars because of the relative weakness of the photospheric background, when they would have been classified as normal flares on the Sun because of its much higher photospheric background.

Spectral emission lines are the most appropriate diagnostics to constrain the physical properties and motions of the flare plasmas. Unfortunately, only a few sets of observations with adequate time and spectral resolution are so far available (e.g. Rodonó et al. 1989; Hawley \& Pettersen 1991; Houdebine 1992a; Gunn et al. 1994; Garcia-Alvarez et al. 2001). Furthermore, there are also few attempts to derive the physical parameters of the flare plasmas (e.g. Katsova 1990; Bruevich et al. 1990; Katsova et al. 1991; Houdebine et al. 1991; Houdebine et al. 1993a, 1993b; Gunn et al. 1994; Jevremovic et al. 1998; Garcia-Alvarez et al. 2001) or the magnetic field topology and localisation of the flare components (Houdebine et al. 1993b). Good quality spectroscopy and a correct description of the different flare components (kernels, prominences and so on) are required to constrain numerical simulations. Besides, each flare is a unique event that may depart a lot from the average flare properties, and one must combine average properties to flare details in order to constain the relevant models.

Here we aim to extract the general characteristics of flares from a compilation of medium resolution spectroscopic observations in the $3600 \AA-4400 \AA$ wavelength range. Below, we compile all available spectroscopic data of stellar flares in order to obtain empirical correlations. We emphasize that an appropriate time resolution is also required, to allow at least for the distinction in the main flare phases.

\section{Flux time profiles}

Flares exhibit quite different spectroscopic behaviour from one flare to another; some evolve on time scales of tens of seconds whereas others may last for a day or more (Katsova et al. 1999; Foing et al. 1994). First, one should distinguish between solar-type flares that evolve typically on time scales shorter than a few tens of minutes and "RS CVn" type flares that last typically more than a couple of hours (Baliunas et al. 1984;
Linsky et al. 1989; Foing et al. 1994). Although there is most likely a continuity between the two classes of flares, it is important to differentiate them because they are very different in their spectroscopic signatures and physical properties. As was proposed by Simon et al. (1980), RS CVn flares may involve magnetic fields from the two stars in the system, leading to flares with a somewhat different nature. In this study, we will deal with solar-type flares only.

Solar-type flares on stars exhibit a variety of behaviour, but there are emerging patterns that seem to apply to most events. For instance, some authors (e.g. Houdebine 1992a) found that the solar description of the flare as a succession of an impulsive phase and a gradual phase usually holds. To illustrate this, we show three flares in Fig. 1, as observed in the $\mathrm{H} \gamma$ line, the Ca II K line and the continuum at about $4200 \AA$. At this wavelength, there are no spectral lines and the Balmer line wing contribution is minimal.

As reported by previous authors (e.g. Rodonó et al. 1989), the white light and $\mathrm{Ca}$ II $\mathrm{K}$ line best describe respectively the impulsive phase and the gradual phase. On the contrary, the Balmer lines may appear in various proportions during the two phases, particularly the gradual phase when they may be weak.

In Fig. 1 top panel, the 8:50 h UT flare on Prox Cen is dominated by a rapid rise (less than $60 \mathrm{~s}$ ) and decay (1 to $2 \mathrm{~min}$ ) in the continuum and the Balmer lines. A slower gradual phase is visible in the $\mathrm{Ca}$ II $\mathrm{K}$ line but not in the Balmer lines. This flare (PC1 thereafter) could be qualified as being mostly "impulsive" in character. The second flare, at 8:21 h UT on the same day (PC2 thereafter), displays no detectable white light emission and the rise and fall in the line fluxes are slow, typical of a solar or stellar flare gradual phase. Such a flare is "gradual" in character and is in fact the closest equivalent to the classical solar flare. Note that for those two flares on Proxima Centauri, the maximum line fluxes are almost identical (see Table 4). This shows that the energy release mechanisms behave in a rather different maner for these two flares, in one case the energy release is quite impulsive whereas in the other it is quite gradual. These flares are good examples of how varied the spectroscopic signatures can be. We may add that during the PC1 flare, the Balmer lines showed significant broadening, with a full width half maximum up to $5.2 \AA$ decreasing rapidly, whereas for the PC2 flare they remained narrower and evolved more slowly throughout the flare (Houdebine 1990). As we shall see below, this is a general feature of flares, the impulsive phase of a flare is always accompanied by an important broadening of the Balmer and Her lines whereas the gradual phase is not.

The third flare is a combination of both an impulsive and a gradual phase with comparable magnitudes, where the two phases are well delineated by their respective spectral tracers; the white light and the Balmer lines for the impulsive phase, and the $\mathrm{Ca}$ II and Balmer lines for the gradual phase. This is so far probably one of the best examples of a "combined flare", where the impulsive and gradual phases combine together with similar magnitudes.

We found that these three classes of flares, impulsive, gradual and combined are sufficient to classify all flares with spectroscopy. We list in Table 1 all flares observed spectroscopically (with adequate time resolution) with its 

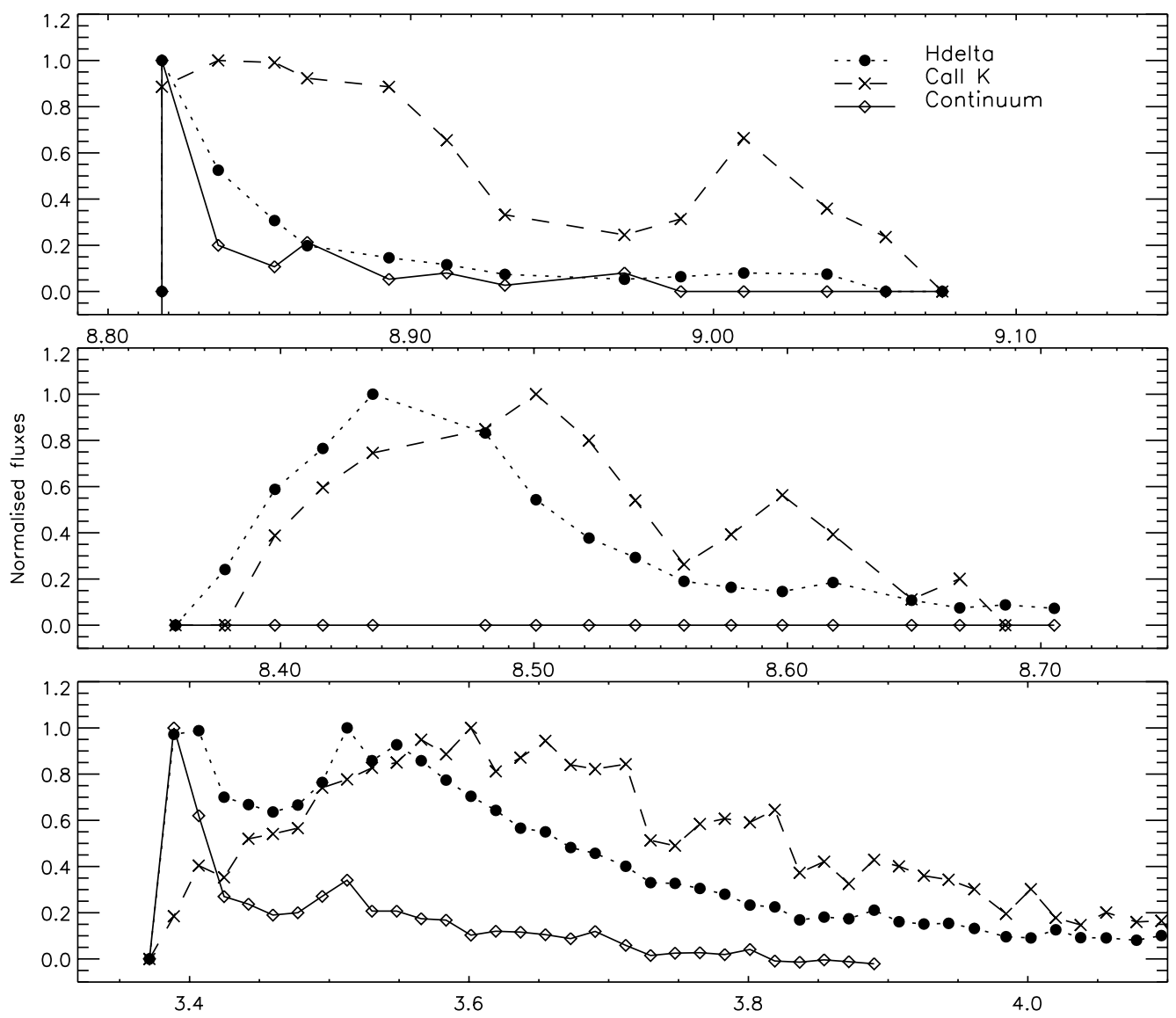

Fig. 1. Flux time profiles in the $\mathrm{H}_{\delta}$ line, Ca II K line, and the continuum at about $4200 \AA$ A for three flares on Proxima Centauri (Houdebine 1992a) and AD Leonis (Rodonó et al. 1989). From top to bottom, the flares started at 8:50 UT, 8:21 UT on 25 March 1984 and 3:22 UT on 28 March 1984 respectively. This diagram, representing solar-like flares, illustrates how varied flare behaviour can be, from totally "impulsive" (upper panel) to totally "gradual" (intermediate panel), or a combination of both (lower panel).

classification according to the above description. We found that "non-white light gradual flares" represent a minority of the events, with $25 \%$. This is in contrast with the solar case where white light flares are relatively rare. This apparent difference may however be due, at least in part, to a contrast effect: we illustrate this point by refering to Fig. 29 in Houdebine (1992a). Light curves for three flares were reported on an absolute scale and compared to the flux emitted in the $U$-band by an area of $10^{19} \mathrm{~cm}^{2}$ on the sun (this is about the area of a white light kernel for a solar flare). Clearly, the weakest 0.3 mag flare on Proxima Centauri would have remained undetected if occuring on the Sun. It is therefore possible that, like the flares detected on stars (Table 1), most solar flares are actually white-light flares with a weak white light emission, and only a minority are "normal" non-white light flares. One should also consider the fact that the $U$-band includes the Balmer recombination continuum and the higher Balmer lines in the series. Hence further studies in spectrophotometry are necessary to determine what proportion of the flares actually emit white light.

Nevertheless, it seems that flares with no white light emission do indeed exist. Such purely non-white light flares seems to have been observed on Proxima Cen (Fig. 1, PC1 flare) and UV Ceti (Butler et al. 2002). They had no detectable white light emission in spectrophotometry even though for the PC1 flare its spectral line emission was fairly strong (8 times the quiescent emission).

We notice that large flares are always combined flares whereas the impulsive flares are always comparatively weak (Tables 1 and 4). The gradual phase in the Balmer lines during flares with an impulsive phase remains undetectable for a $U$ band maximum flux below $\sim 7 \times 10^{29} \mathrm{erg} / \mathrm{s}$. The mechanism responsible for the Balmer line emission during the gradual phase remains unclear, but this mechanism clearly does not operate as efficiently for weak impulsive flares. Another possible explanation is that the two phases become increasingly blended in time when the flare energy diminishes. This would be in agreement with the fact that the rise and decay times in the spectral lines diminish with the flare energy (see Sect. 4). Along the same lines, we notice that small flares are short while large flares may last for tens of minutes (Tables 1 and 4). This is not an observational selection effect. This dependence of the flare duration on flare power will be developed in Sect. 4 .

So far, the stellar flare phenomenology closely resembles that of solar flares. For instance, evolution time scales are very similar; the impulsive and gradual phases respectively last for a few minutes and few tens of minutes. Also, the relative amplitudes of these phases can be similar to that of their solar equivalents: In the solar case, the total emission in $\mathrm{H}_{\alpha}$ from 
Table 1. Main characteristics of solar-like flares on dwarfs as observed in spectroscopy. Note that because of the relatively poor time resolution of most observations $(\geq 1 \mathrm{mn})$, the rise time and decays may be rather uncertain and sometimes only upper limits are measurable. Because of the often poor spectral resolution, a large uncertainty also applies to FWHM measurements, in particular for the Ca II lines. The rise time of the gradual phase is measured from the flare onset.

\begin{tabular}{|c|c|c|c|c|c|c|c|c|c|c|c|c|}
\hline \multirow[t]{3}{*}{ Star } & \multirow[t]{3}{*}{ UT } & \multirow[t]{3}{*}{ Date } & \multirow{2}{*}{\multicolumn{3}{|c|}{$\begin{array}{c}\text { Impulsive Phase } \\
\mathrm{H}_{\mathrm{I}}\end{array}$}} & \multicolumn{5}{|c|}{ Gradual Phase } & \multirow[t]{3}{*}{ Character } & \multirow[t]{3}{*}{ Authors } \\
\hline & & & & & & \multicolumn{3}{|c|}{ HI } & \multicolumn{2}{|c|}{ Ca II } & & \\
\hline & & & $\begin{array}{l}\text { Rise } \\
(\mathrm{mn})\end{array}$ & $\begin{array}{l}\text { Decay } \\
(\mathrm{mn})\end{array}$ & $\begin{array}{c}F W H M \\
(\AA)\end{array}$ & $\begin{array}{l}\text { Rise } \\
(\mathrm{mn})\end{array}$ & $\begin{array}{l}\text { Decay } \\
(\mathrm{mn})\end{array}$ & $\begin{array}{c}F W H M \\
(\AA)\end{array}$ & $\begin{array}{l}\text { Rise } \\
(\mathrm{mn})\end{array}$ & $\begin{array}{l}\text { Decay } \\
(\mathrm{mn})\end{array}$ & & \\
\hline UV Ceti & $11: 19$ & $08 / 09 / 79$ & - & - & - & 12.5 & - & - & 20 & - & Combined? & Eason et al. (1992) \\
\hline Prox Cen & $8: 21$ & $25 / 03 / 84$ & - & - & - & 4.8 & 9.6 & 3.8 & 8.6 & 12.5 & Gradual & Houdebine (1990) \\
\hline Prox Cen & $8: 50$ & $25 / 03 / 84$ & $<1.2$ & 2.8 & 5.5 & - & - & - & 2.2 & 7.2 & Impulsive & Houdebine (1990) \\
\hline AD Leo & $2: 40$ & $28 / 03 / 84$ & 0.65 & $\sim 2.7$ & 10.5 & - & - & - & - & - & Impulsive & Houdebine (1990) \\
\hline AD Leo & $3: 22$ & $28 / 03 / 84$ & 0.5 & $\sim 3$ & 9.9 & 9.7 & 18.7 & 4.9 & 13.9 & 29.1 & Combined & Rodonó et al. (1989) \\
\hline UV Ceti & $1: 53$ & $06 / 12 / 84$ & - & - & - & 1.8 & 3.3 & - & 3.0 & 6.0 & Gradual & Butler et al. $(1986,2002)$ \\
\hline UV Ceti & $2: 03$ & $06 / 12 / 84$ & - & - & - & 2.4 & 6.0 & - & - & - & Gradual & Butler et al. $(1986,2002)$ \\
\hline UV Ceti & $2: 29$ & $06 / 12 / 84$ & - & - & - & 2.1 & 3.7 & - & 5.2 & 8.5 & Gradual & Butler et al. $(1986,2002)$ \\
\hline UV Ceti & $2: 43$ & $06 / 12 / 84$ & - & - & - & 4.6 & 6.0 & - & 8.0 & 19.6 & Impulsive & Butler et al. $(1986,2002)$ \\
\hline UV Ceti & $3: 16$ & $06 / 12 / 84$ & - & - & - & 1.8 & 3.3 & - & 1.8 & 4.5 & Gradual & Butler et al. $(1986,2002)$ \\
\hline UV Ceti & $3: 29$ & $06 / 12 / 84$ & 1.9 & 7.7 & - & - & - & - & 8.3 & 16.6 & Impulsive & Butler et al. $(1986,2002)$ \\
\hline YZ CMi & $19: 55$ & $04 / 03 / 85$ & 2.3 & 5.5 & 10.5 & - & - & - & - & - & Impulsive & Doyle et al. (1988) \\
\hline AD Leo & $4: 40$ & $12 / 04 / 85$ & 10 & - & 4.9 & 19.6 & 34.6 & 4.5 & 29.4 & 75.8 & Combined & Hawley \& Pettersen (1991) \\
\hline AT Mic & $1: 12$ & $20 / 08 / 85$ & 2.5 & - & 11.9 & 7.7 & 25.7 & 7.3 & 11.5 & 31.6 & Combined & Garcia-Alvarez et al. (2001) \\
\hline $\mathrm{T} 48$ & 13:00 & $04 / 12 / 85$ & - & - & - & 48 & 114 & 5 & 81 & 127 & Combined? & Carter et al. (1988) \\
\hline II Peg & $2: 10$ & $17 / 08 / 89$ & 8.8 & - & 8.5 & 33.4 & 70.3 & 6.8 & 51 & 97 & Combined & Doyle et al. (1992) \\
\hline G1 866 & $3: 27$ & $11 / 06 / 91$ & 1.7 & - & - & - & - & - & - & - & Impulsive & Jevremović et al. (1998) \\
\hline G1 866 & $3: 56$ & $11 / 06 / 91$ & 1.3 & 2.2 & - & - & - & - & - & - & Impulsive & Jevremović et al. (1998) \\
\hline Gl 866 & $4: 24$ & $11 / 06 / 91$ & 1.5 & 6.2 & - & - & - & - & - & - & Impulsive & Jevremović et al. (1998) \\
\hline AD Leo & $0: 45$ & $14 / 03 / 98$ & 0.29 & 0.68 & 4.4 & 4.5 & 7.1 & 3.2 & 0.38 & 0.59 & Combined & Jevremović (1999) \\
\hline
\end{tabular}

the kernels is about the same as for the ribbons (extended areas) (Tandberg-Hanssen \& Emslie 1988), which compares to the combined flares.

To conclude, we found that the spectral line and $U$-band time profiles obey well defined patterns except for "complex" flares when more than one $U$-band event occur. From the observations gathered so far we extracted the following properties, some of which will be developed in the next section:

a Impulsive phase: Balmer and $\mathrm{He}$ I lines are time and flux correlated to the white light, and evolve rapidly;

b Impulsive phase: Balmer and He I lines decrease slower than the white light;

c Impulsive phase: Lower chromospheric lines (e.g. $\mathrm{Ca}$ / $\mathrm{Ca}$ II, $\mathrm{Mg}$ II) are most often very weak and evolve slowly;

d Gradual phase: He I line is weak or absent, Balmer lines are usually strong for medium and large flares but may be absent in small flares;

e Gradual phase: Lower chromospheric lines are strong and evolve slowly;

f During both phases the time scales of evolution (rise and decay) seem to depend on the temperature of formation of the line, i.e. higher temperature lines evolve faster.

\section{Time lags in the flux time profiles}

We compiled the rise and decay times in the $\mathrm{Ca}$ II $\mathrm{K}$ and $\mathrm{H}_{\gamma}$ lines for all flares where this was possible (Table 1). The rise time is the time lag between flare onset and the time of maximum flux, and the decay time is the time lag between maximum flux and the time when the line has decayed to half of this value. We plot in Fig. 2 some of these delays. We found good correlations between all of the rise and decay times except with the rise and decay times of $\mathrm{H}_{\gamma}$ for the impulsive phase. This is due to the fact that the impulsive phase is not well resolved, i.e. rise and decay times are typically of the order of one minute or less and the time resolution is also about one minute in the best cases (except for the AD Leo flare observed by Jevremović 1999).

The correlation between the $\mathrm{Ca}$ II $\mathrm{K}$ and $\mathrm{H}_{\gamma}$ rise times is excellent (Fig. 2a). Measurements do not depart from the correlation by more than $2.5 \mathrm{~min}$. This figure also emphasises how varied the flares can be, with rise times from less than 2 min to more than $80 \mathrm{~min}$. Furthermore, it seems that there are no preferred time scales, and the distribution seems to reflect the flare energy distribution - i.e. - small flares are more frequent and evolve on smaller time scales (see Sect. 4). We give in Table 2 the parameters for the best linear fit to the data. From this correlation, we find that the $\mathrm{Ca}$ II $\mathrm{K}$ line is systematically 1.63 times slower to rise than the $\mathrm{H}_{\gamma}$ line.

The correlation between the rise and decay times in the $\mathrm{H}_{\gamma}$ Balmer line is also good (Fig. 2b). This line is typically 2.3 times slower to decay than to rise. The $\mathrm{Ca}$ II $\mathrm{K}$ line decay seems to depart from this linear behaviour (Figs. 2c and 2d). This line is very slow to decay, and we found that the correlations were best fitted by a second degree polynomial function. We find that for small flares the decay time is of the order of twice the rise time whereas for larger flares this factor is of the order of 1.8. We found a similar result for the decay time in $\mathrm{H}_{\gamma}$ as a function of the decay time in Ca II K (Fig. 2d). For large flares the $\mathrm{H}_{\gamma}$ and $\mathrm{Ca}$ II decay times are more similar whereas for smaller flares the decay time in $\mathrm{Ca}$ II $\mathrm{K}$ is a factor of two larger than that in $\mathrm{H}_{\gamma}$. 

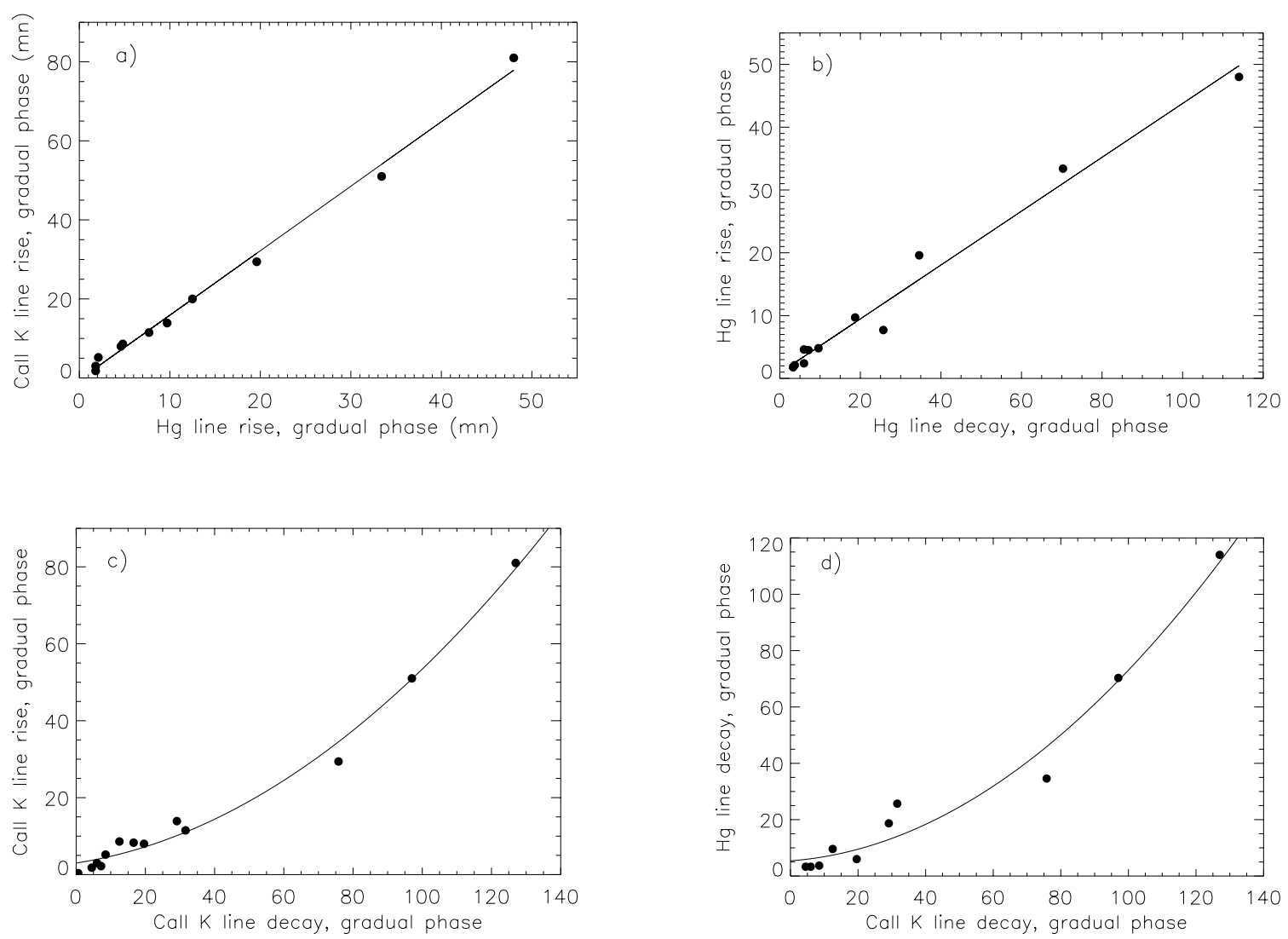

Fig. 2. Rise and decay times in the $\mathrm{Ca}$ II $\mathrm{K}$ and $\mathrm{H}_{\gamma}$ lines. We find good correlations between these times a) to d) that provide valuable constraints on the properties of the plasmas. These diagrams show that all flares evolve along the same pattern in time, i.e. the Ca II $\mathrm{K}$ line is always 1.63 times slower to rise.

The rise and decay times obey good relationships which signifies that there is a well defined underlying mechanism responsible for the flux time profiles in these lines during the gradual phase. Such a mechanism necessarily involves the natural cooling of the flare plasmas if we assume that most of the energy release occurs during the impulsive phase and that little heating takes place during the gradual phase. We must then center our interpretation on how the cooling of the flare plasma can produce the observed features. Actually, models for the cooling of the plasma (Gurzadyan 1984) and calculations of the cooling curves from the observed time profiles (Houdebine et al. 1991) show that it is very easy to reproduce the observed line time profiles by the natural cooling of a flare plasma. In this context, the rise and decay times depend on the density of the plasma, and the longer these delays the smaller is the density (Gurzadyan 1984). According to Gurzadyan calculations (Gurzadyan 1984, Fig. 10), the plasma density $n$ is related to the rise time $t_{\text {Caii }}^{\text {rise }}$ in the $\mathrm{Ca}$ II $\mathrm{K}$ line according to the following relationship;

$\log (n) \simeq-0.9917 \log \left(t_{\text {Caii }}^{\text {rise }}\right)+14.528$

where $t_{\text {Caii }}^{\text {rise }}$ is in s and $n$ in $\mathrm{cm}^{-3}$. This gives a total density of $\sim 5.8 \times 10^{12} \mathrm{~cm}^{-3}$ for a time of $2 \mathrm{mn}$ and a density of $\sim 7.5 \times 10^{10} \mathrm{~cm}^{-3}$ for a time of $80 \mathrm{mn}$. Now, according to model chromospheres of dMe stars (Houdebine \& Doyle 1994a,b; Houdebine \& Stempels 1997) the total density is of the order of $1-5 \times 10^{12} \mathrm{~cm}^{-3}$ in the quiescent chromosphere of active dwarfs. Furthermore, if additional heating has taken place during the flare, the density is likely to be larger than the above quiescent chromospheric value. This means that, except for the fastest flares, the flare plasma is unlikely to be located in the chromosphere. Instead, the plasma is rather a low density and cool coronal plasma.

Figure 2a shows that the delays may vary over more than one order of magnitude. As mentioned above, this implies that the coronal plasmas have densities varying over almost two orders of magnitude. The other correlations found between the rise and decay times and the radiated energies (Figs. 4a to $4 \mathrm{c}$, see Sect. 4) are therefore evidence that large flares have lower densities than small flares. This result will be further discussed in Sect. 4. Another result that supports the cooling model is the fact that the rise and decay times in the Ca In line are usually longer than the rise and decay times in the Balmer lines (Figs. 2a and 2d). This result is expected since the gradient in the cooling curve diminishes with time and temperature (Gurzadyan 1984; Houdebine et al. 1991). In other words radiative cooling efficiency diminishes with temperature, and therefore with time. This behaviour shows that high temperature lines evolve faster than low temperature lines, and agrees with numerical models (Gurzadyan 1984).

Besides the radiative cooling, another competing mechanism that could play a role in the line time profiles during the gradual phase is X-ray backwarming. Hawley \& Fisher (1992) calculated the spectra resulting from X-ray backwarming, for 
a series of 5 models. Their models can reproduce Balmer line fluxes. However, they fail to reproduce the $\mathrm{Ca}$ II to Balmer line ratios and the continuum. So far, with the present calculations it is not clear whether X-ray backwarming can explain the observed time-profiles in both the Balmer and Ca II lines, and in particular the differences in the time delays we report here. Further calculations are required to find out if X-ray backwarming can play a role in the correlations observed in Figs. 2 a to $2 \mathrm{~d}$.

\section{Maximum fluxes in the line and continuum time profiles}

\subsection{Absolute line and continuum fluxes}

Absolute fluxes for the continuum or spectral lines were commonly derived using model photospheric calculations such as those of Mould (1976) combined with main-sequence spectral type-luminosity empirical correlations (e.g. Veeder 1974). However, both are actually rather inaccurate. The former because until recently (e.g. Allard \& Hauschildt 1995) they lacked adequate opacity tables and the latter because the mainsequence at spectral type $M$ spreads over three magnitudes and active dwarfs are often over-luminous by $1.5 \mathrm{mag}$ or so (e.g. Mirzoyan 1993). This could yield fluxes under-estimated by a factor of 4 to 5 depending on the star's absolute magnitude.

Here we calculate absolute fluxes from the measured parallax. We compile the data adopted in this study in Table 3. The data was taken from the Hipparcos and Tycho catalogues (Turon et al. 1997), the new Gliese Catalogue (1991) or Strassmeier et al. (1993). $R-I$ data is from Leggett (1992) or Gliese $(1969,1991)$ and homogenised to the Kron system using Legget (1992) transformations. The effective temperatures were calculated according to the empirical effective temperature relationship of Jones et al. (1994). The $U$-band absolute fluxes were computed assuming a flux of $4.35 \times 10^{-9} \mathrm{erg} / \mathrm{cm}^{2} / \AA$ at Earth for a 0 mag star (Johnson 1966), and assuming an effective $U$-band width of $680 \AA$ (Allen 1973).

\subsection{Maximum fluxes}

We measured the maximum fluxes in the $U$-band, the $\mathrm{H}_{\gamma}$ and the $\mathrm{Ca}$ II $\mathrm{K}$ lines during the impulsive and the gradual phases for all flares for which the relevant time profiles were available. We list the results in Table 4 . We plotted the $\mathrm{H}_{\gamma}$ flux during the impulsive phase against the $U$-band flux in Fig. 3a. We find a good correlation that does not seem to be linear in a log-log scale. We fitted the correlation with a second order polynomial function (Table 2). For large flares the slope of the observed correlation is close to 1 , i.e., the $\mathrm{H}_{\gamma}$ flux grows as fast as the $U$-band flux, but for small flares, the slope is of the order of 0.34 which means that the $U$-band flux is growing three times faster than the $\mathrm{H}_{\gamma}$ flux.

Houdebine (1992a, H92a thereafter) found a similar correlation between the $\mathrm{H}_{\delta}$ line flux and the continuum flux at $4200 \AA$ during the impulsive phase for four flares. The difference between the present correlation and the one in $\mathrm{H} 92 \mathrm{a}$ is mainly that the present correlation covers 5.5 orders of magnitude whereas we had 3.5 orders of magnitude in H92a.
Also, the present correlation only includes the fluxes measured at flare maximum. This correlation includes higher energy flares than in H92a and it can be approximated by a linear relationship in the $5 \times 10^{26}-10^{32}$ ergs energy range, which explains why H92a found a linear correlation.

The significant difference mentioned above between the gradient for low and high energy flares leads to important constraints on the properties of the impulsive phase. H92a showed that most of the spectral signatures during this phase could be reproduced by two series of models: the models for the dense flare kernels, and the models for the radiative pumping of the Balmer emission by white light. But these models give a slope that is in agreement with the correlation found in H92a between the $\mathrm{H}_{\delta}$ flux and the continuum flux, for which the slope is 0.61 .

However, the models presented in H92a for the radiative pumping study are based on a relatively low pressure model chromosphere. Houdebine (1990, H90 thereafter) studied the radiative pumping phenomenon in the Balmer lines for two quiescent model atmospheres that are quite different in order to evaluate the amplitude of this effect in very different atmospheric conditions. $\mathrm{H} 90$ found that the chromospheric response to the white light irradiation was significantly different for the two models. For the low pressure model atmosphere (series of models M.A.1 in H90) the gradient of the growth of $\mathrm{H}_{\delta}$ viz. $U$-band flux is of the order of 0.6 whereas for the high pressure model atmosphere, this gradient is $\sim 1.7$. Houdebine \& Doyle (1994a) also investigated these model chromospheres and showed that low pressure models (such as M.A.1 in H90) are inadequate. It is therefore more likely that real effect of radiative pumping on the atmosphere will be a large gradient of 1 or more. This means that radiative pumping is able to reproduce the larger gradient observed for large flares (Fig. 3a).

To further illustrate the effect of radiative pumping on the Balmer lines, we plot the $\mathrm{H}_{\gamma}$ line profiles in Fig. 4 for the M.A. 2 models in H90. 23 models were computed with increasing Balmer continuum irradiation. Model 1 corresponds to the quiescent atmosphere with no irradiation. The Balmer continuum irradiation increases from model 1 to model 23. The resulting effect is essentially to increase the line flux, because the Balmer continuum radiation ionises hydrogen atoms, depletes the level 2 population, and as a consequence depletes the Balmer line optical depths and allows the atmosphere to radiate more in the Balmer lines. Some Balmer line profiles also become narrower, and in particular $\mathrm{H}_{\alpha}$ (see H90). The selfreversal in $\mathrm{H}_{\alpha}$ diminishes and eventually disapears (see H90). For further comparison with the present observations, we plot in Fig. 5 the flux in the Balmer series as a function of the flux in the Balmer continuum at threshold for the M.A. 2 models in H90. The effect of the Balmer continuum on the Balmer lines is rather homogeneous along the series, and the variations are almost linear in a log-log scale. These curves can be compared to the correlation in Fig. 3a, providing one multiplies the theoretical fluxes by a given area.

In any case, the kernel models (H92a) seem unable to produce a Balmer line emission that increases as found in our correlation, i.e., a gradient that increases from small flares to large flares. Instead, in these models, increasing white light radiation implies increasing pressure and opacity in the atmosphere. 
Table 2. Empirical correlations between the various flare spectroscopic parameters. We give the parameters and correlation coefficient or $\chi^{2}$ for each correlation as well as the function that was fitted to the data.

\begin{tabular}{|c|c|c|c|c|c|}
\hline Correlation: $x, y$ & $a$ & $b$ & $c$ & $\begin{array}{l}\text { Cor. Coef. } \\
\text { or } \chi^{2} \\
\end{array}$ & Function $y=f(x)$ \\
\hline $\begin{array}{l}\mathrm{H}_{\gamma} \text { rise, grad./ } \\
\text { Ca II K rise, grad. }\end{array}$ & 1.63 & -0.425 & - & 0.997 & $y=a \cdot x+b$ \\
\hline $\begin{array}{l}\mathrm{H}_{\gamma} \text { decay, grad./ } \\
\mathrm{H}_{\gamma} \text { rise, grad./ }\end{array}$ & 0.429 & 0.899 & - & 0.990 & $y=a \cdot x+b$ \\
\hline $\begin{array}{l}\mathrm{Ca} \text { II } \mathrm{K} \text { decay, grad./ } \\
\mathrm{Ca} \text { II } \mathrm{K} \text { rise, grad. }\end{array}$ & $3.66 \mathrm{E}-3$ & 0.138 & 3.02 & 7.46 & $y=a \cdot x^{2}+b \cdot x+c$ \\
\hline $\begin{array}{l}\text { Ca II K decay, grad./ } \\
\mathrm{H}_{\gamma} \text { decay, grad. }\end{array}$ & $5.88 \mathrm{E}-3$ & 8.9E-2 & 5.40 & 49.0 & $y=a \cdot x^{2}+b \cdot x+c$ \\
\hline $\begin{array}{l}U \text {-band max. flux / } \\
\mathrm{H}_{\gamma} \text { max. flux, imp. }\end{array}$ & 0.114 & -5.90 & 103 & $8.3 \mathrm{E}-2$ & $\log (y)=a \cdot[\log (x)]^{2}+b \cdot \log (x)+c$ \\
\hline $\begin{array}{l}U \text {-band max. flux / } \\
\text { Ca II K max. flux }\end{array}$ & 0.130 & -6.87 & 116 & $2.2 \mathrm{E}-1$ & $\log (y)=a \cdot[\log (x)]^{2}+b \cdot \log (x)+c$ \\
\hline $\begin{array}{l}U \text {-band max. flux / } \\
\mathrm{H}_{\gamma} \text { max. flux, grad. }\end{array}$ & 0.986 & -1.55 & - & 0.971 & $\log (y)=a \cdot \log (x)+b$ \\
\hline $\begin{array}{l}\mathrm{H}_{\gamma} \text { max. flux, grad/ } \\
\text { Ca II K max. flux }\end{array}$ & 0.941 & 1.24 & - & 0.990 & $\log (y)=a \cdot \log (x)+b$ \\
\hline $\begin{array}{l}\mathrm{H}_{\gamma} \text { max. flux, imp/ } \\
\mathrm{H}_{\gamma} \text { max. flux, grad. }\end{array}$ & 0.932 & 1.85 & - & 0.963 & $\log (y)=a \cdot \log (x)+b$ \\
\hline $\begin{array}{l}U \text {-band max. flux / } \\
\text { Ca II K rise, grad. }\end{array}$ & 2.169 & -119 & 1630 & 26.5 & $y=a \cdot[\log (x)]^{2}+b \cdot \log (x)+c$ \\
\hline $\begin{array}{l}\text { Ca II K decay, grad./ } \\
\text { Ca II K max. flux }\end{array}$ & $4.38 \mathrm{E}-2$ & 25.5 & - & 0.890 & $\log (y)=a \cdot x+b$ \\
\hline $\begin{array}{l}\text { Ca II K rise, grad. / } \\
\mathrm{H}_{\gamma} \text { max. flux, grad. }\end{array}$ & $7.85 \mathrm{E}-2$ & 26.3 & - & 0.826 & $\log (y)=a \cdot x+b$ \\
\hline $\begin{array}{l}U \text {-band flux / } \\
\mathrm{H}_{\delta} \text { flux }\end{array}$ & 0.615 & 11.6 & - & 0.921 & $\log (y)=a \cdot \log (x)+b$ \\
\hline
\end{tabular}

This increased opacity tends to diminish the Balmer line intensity relative to the continuum intensity, as the emission tends towards that of a black-body. In other words the Balmer line equivalent widths should decrease with white light intensity for high values, and not increase as is observed. Therefore, emission from the kernels, even though it reproduces well the observed correlation for medium size flares (H92a), cannot explain the entire correlation.

Hence, the only way to explain both a varying gradient and a gradient of 1 for large flares (Fig. 3a) is to speculate that radiative pumping/backwarming represents a significant proportion of the total Balmer emission during large flares and that this proportion increases with the flare energy. Therefore, observations indicate that, for some as yet unknown reason, radiative pumping in the surrounding atmosphere and probably also backwarming by white light becomes more efficient as the flare energy increases. We speculate that this may have something to do with the height of the white light source in the atmosphere: if the source is at low atmospheric levels, it will radiate radially into the surrounding quiescent chromosphere and will affect only a small area around the flare (because the large chromospheric opacity prevents the radiation from propagating very far), while if it is located higher in the corona the area in view will be considerably larger. Observations indicate that this source of white light increases in height, or extends further in height, as the flare energy increases.

This interpretation finds additional support in the line FWHM observations: if the flare kernels were the dominant source of radiation during the impulsive phase, the Balmer line widths should be large for large flares as one expects a larger heating to take place. Instead, the line widths seem to increase till a maximum attained for a $U$-band energy of a few times 
Table 3. We give the photometric data, absolute magnitude and its uncertainty due to the parallax error, effective temperature derived from the $(R-I)_{K}$ color, the absolute flux conversion factor and the absolute flux in the $U$-band for the stars on which flares were observed.

\begin{tabular}{|c|c|c|c|c|c|c|c|c|c|c|}
\hline Star & Gl No & $\begin{array}{c}V \\
(\mathrm{mag}) \\
\end{array}$ & $\begin{array}{l}U-B \\
(\mathrm{mag}) \\
\end{array}$ & $\begin{array}{l}B-V \\
(\mathrm{mag}) \\
\end{array}$ & $\begin{array}{l}R-I \\
(\mathrm{mag}) \\
\end{array}$ & $\begin{array}{c}\pi \\
\left(\mathrm{m}^{\prime \prime}\right) \\
\end{array}$ & $\begin{array}{c}M_{V} \\
(\mathrm{mag}) \\
\end{array}$ & $\begin{array}{l}T_{\text {eff }} \\
(\mathrm{K}) \\
\end{array}$ & $\begin{array}{c}F_{U} \\
\left(\operatorname{erg~s}^{-1}\right) \\
\end{array}$ & Factor \\
\hline \multirow[t]{2}{*}{ UV Ceti } & $65 \mathrm{~A}$ & 12.57 & 1.10 & 1.85 & 1.64 & 3814 & 15.470 .02 & 2735 & $1.5 \mathrm{E} 27$ & 8.24 E38 \\
\hline & $65 \mathrm{~B}$ & 12.70 & - & - & - & $"$ & 15.600 .02 & - & $1.3 \mathrm{E} 27$ & $"$ \\
\hline YZ CMi & 285 & 11.4 & 1.00 & 1.60 & 1.34 & 1634 & 12.460 .05 & 3023 & 3.3 E28 & $4.50 \mathrm{E} 39$ \\
\hline AD Leo & 388 & 9.40 & 1.08 & 1.54 & 1.12 & 2043 & 10.950 .03 & 3234 & 1.3 E29 & $2.88 \mathrm{E} 39$ \\
\hline \multirow[t]{2}{*}{ FL Vir } & $473 \mathrm{~A}$ & 13.04 & 1.20 & 1.83 & 1.58 & 2324 & 14.870 .04 & 2793 & 2.4 E27 & 2.22 E39 \\
\hline & $473 B$ & 13.30 & - & - & - & $"$ & 15.100 .04 & - & $1.9 \mathrm{E} 27$ & , \\
\hline Prox Cen & 551 & 11.1 & 1.43 & 1.83 & 1.60 & 7625 & 15.510 .01 & 2774 & $1.1 \mathrm{E} 27$ & $2.06 \mathrm{E} 38$ \\
\hline BY Dra & 719 & 8.48 & 1.04 & 0.97 & - & 586 & 7.290 .21 & - & 6.7 E30 & $3.56 \mathrm{E} 40$ \\
\hline AT Mic & 799A & 10.2 & 0.88 & 1.57 & 1.33 & 1176 & 10.540 .11 & 3033 & 2.3 E29 & 8.74 E39 \\
\hline AU Mic & 803 & 8.96 & 0.95 & 1.47 & 0.85 & 1036 & 9.020 .12 & 3493 & 9.4 E29 & $1.13 \mathrm{E} 40$ \\
\hline EV Lac & 873 & 10.26 & - & 1.61 & 1.20 & 1974 & 11.730 .04 & 3157 & - & $3.08 \mathrm{E} 39$ \\
\hline G1 866 & 866 & 12.33 & 1.54 & 1.96 & 2.04 & 3059 & 14.750 .06 & 2350 & $1.8 \mathrm{E} 27$ & $1.29 \mathrm{E} 39$ \\
\hline \multirow[t]{2}{*}{ EQ Peg } & $896 \mathrm{~A}$ & 10.38 & 1.08 & 1.54 & 1.13 & 1539 & 11.300 .12 & 3224 & 9.6 E28 & 5.11 E39 \\
\hline & 896B & 12.40 & 1.12 & 1.65 & 1.62 & $"$ & 13.320 .12 & 2750 & $8.9 \mathrm{E} 27$ & $"$ \\
\hline $\mathrm{T} 48$ & - & 16.31 & - & 1.07 & 1.12 & $470 \mathrm{pc}$ & 7.95 & 3240 & - & $2.64 \mathrm{E} 43$ \\
\hline II Peg & - & 7.5 & 0.66 & 1.01 & 0.29 & - & 5.4 & $\mathrm{~K} 2.5 \mathrm{~V}-\mathrm{IV}$ & 6.5 E31 & $1.03 \mathrm{E} 41$ \\
\hline
\end{tabular}

$10^{26} \mathrm{erg} / \mathrm{A}$ and decrease for larger flares (see Fig. 7 and H92a). Indeed, for very large flares, surprisingly, line broadening was never observed to be very large: in Hawley \& Pettersen (1991) the maximum line widths are of the order of $5 \AA$. Similarly during the large flares on II Peg (Doyle et al. 1992) and T48 (Carter et al. 1988) a $F W H M$ of only $\sim 5.5 \AA$ was observed. Moreover, H92a observed two components in the Balmer line profiles during the flare on AD Leonis (Table 1). These two components seem to be respectively the signature of the flare kernels and the flare radiative pumping/backwarming of the surrounding chromosphere. Therefore, the variation of the line broadening (see Sect. 5) as a function of $U$-band energy supports the interpretation that the two Balmer line emission mechanisms, kernels and radiative pumping, compete to produce the varying gradient in Fig. 3a.

Another part of the correlation that is difficult to explain is that for $U$-band energies below $10^{28} \mathrm{erg} / \mathrm{s}$. In this energy range, the gradient is only 0.34 . We believe this occurs because the $U$-band is no longer a good tracer of the total flare radiated energy: the evidence in Sect. 2 that there exists small flares with no detectable white light signature (Fig. 1 and Table 1), proves that white light is not a good diagnostic of the total radiated energy budget. In fact, so little is known about the energy radiated accross the spectrum that we don't yet know what spectral diagnostic could be used as a measure of the radiated energy budget. There are some correlations between the fluxes emitted at different wavelengths (e.g. Butler 1993; Benz \& Gudel 1994) but none that involve the total radiated energy budget. We believe that for low energies, the white light emission is not correlated anymore with other spectral diagnostics, and this is the reason why the $U$-band energy varies over more than an order of magnitude while the Balmer lines show little variation (Fig. 3a). For a given spectral line energy the $U$-band flux may span the entire flux range from 0 to $\sim 10^{28} \mathrm{erg}$. In other words, we believe that the $U$-band is a good tracer of the entire flare energy only for a $U$-band peak flux greater than $\sim 10^{28}$ ergs. In Fig. 3a we may therefore distinguish three regions: (i) low energy flares ( $U$-band flux below $10^{28} \mathrm{ergs}$ ) for which the $U$-band is not a good tracer of the energy released, (ii) medium energy flares for which the gradient can be explained by flare kernel models, and (iii) high energy flares for which the gradient increases, and for which the Balmer emission is increasingly dominated by radiative pumping.

We show in Fig. $3 \mathrm{~b}$ the $\mathrm{Ca}$ II $\mathrm{K}$ line flux as a function of the $U$-band flux. The $\mathrm{Ca}$ II $\mathrm{K}$ line flux increases as the $U$-band flux increases but more slowly. For a five orders of magnitude increase in the $U$-band flux, the $\mathrm{Ca}$ II $\mathrm{K}$ line flux increased by only 3.5 orders of magnitude. However, as already mentioned above for the Balmer line- $U$-band correlation, this may be due to small flares: for small flares the spectral line fluxes show little variation for a change of one order of magnitude in the $U$-band. This is not the case for medium and large flares where there is a one to one correlation between the line flux and the $U$ band flux. As already reported above, we believe this occurs for small flares because the $U$-band is not a good tracer of the flare energy. Above $10^{28} \mathrm{erg}$ the $\mathrm{Ca}$ II $\mathrm{K}$ line flux seems to increase with the $U$-band flux with a slope close to one. This means that the magnitude of the gradual phase is directly proportional to the flare energy input.

The $\mathrm{H}_{\gamma}$ line flux during the gradual phase as a function of the $U$-band flux is shown in Fig. 3c. Like the $\mathrm{Ca}$ II $\mathrm{K}$ line for medium and large flares, we find a gradient of $\sim 0.99$, very close to one. Again, this shows that the magnitude of the gradual phase is directly proportional to that of the impulsive phase. This proves that the flare plasmas during the gradual phase are linearly related to the energy released during the impulsive phase. How this plasma is produced and related to the energy release is unknown at this stage, but observations indicate that it has a coronal nature (see Sect. 3).

Figure $3 \mathrm{~d}$ shows the $\mathrm{Ca}$ II $\mathrm{K}$ line flux during the gradual phase as a function of the $\mathrm{H}_{\gamma}$ line flux during this same phase. They are linearly correlated and the slope of the correlation is 

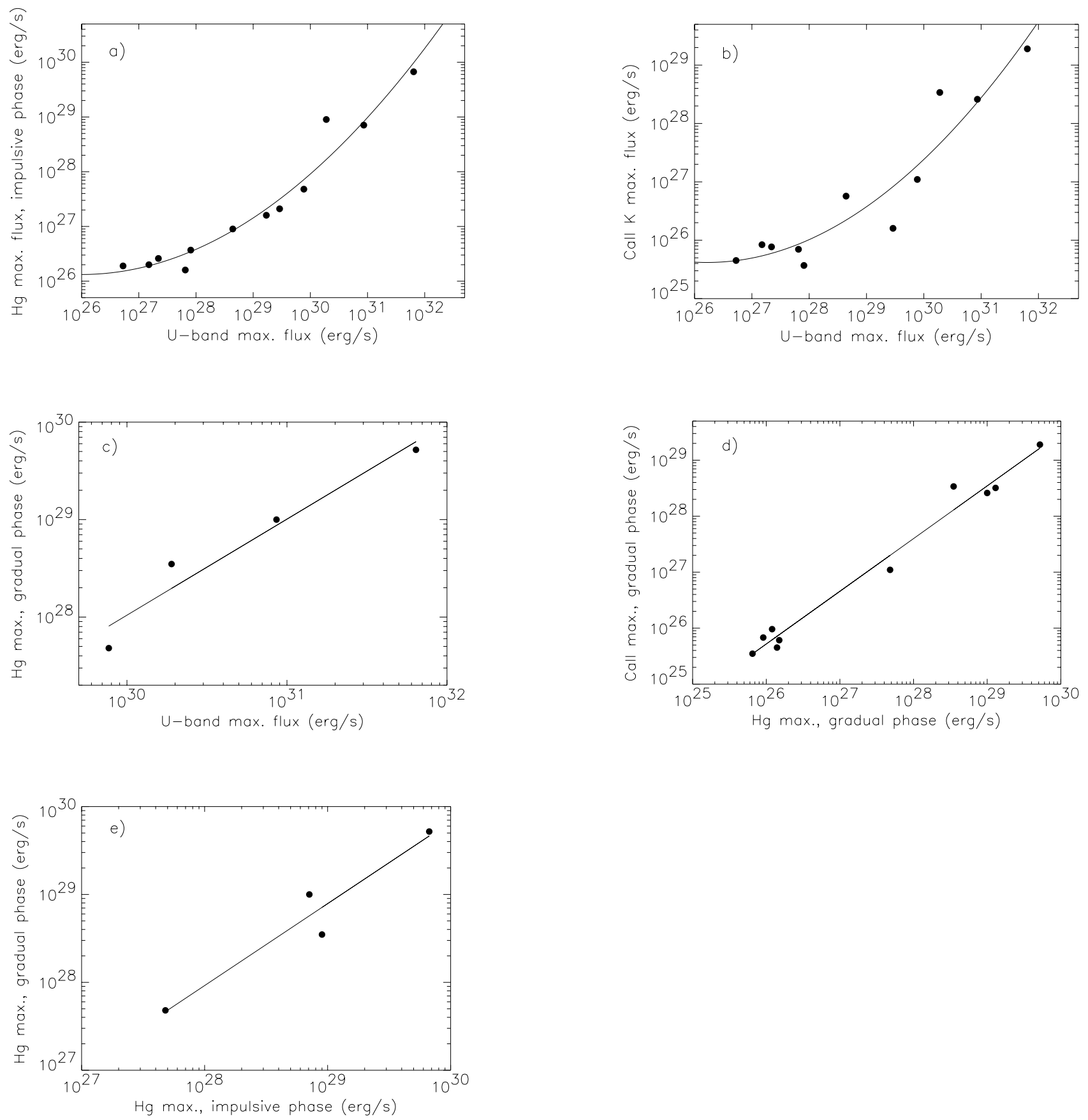

Fig. 3. We plot from a) to e) the $U$-band and line fluxes measured at maximum emission. We obtain a good correlation between the $U$-band and the $\mathrm{H}_{\gamma}$ flux during the impulsive phase (3a). We believe the correlation is evidence that both the emission from dense flare kernels and due to the radiative pumping of the quiescent chromosphere (see text) dominate the Balmer emission during this phase. Other correlations show a one to one linear dependence, which demonstrate that emission lines during the gradual phase scale as the energy released during the impulsive phase.

0.941 (Table 2), which shows again that both the $\mathrm{H}_{\gamma}$ and the Ca II $\mathrm{K}$ lines grow linearly together. On average, according to the correlation (Table 2), the ratio of the $\mathrm{H}_{\gamma}$ line to the $\mathrm{Ca}$ II $\mathrm{K}$ line is about 2.6. This means that the ratio of the amounts of plasma emitting in the Balmer lines and the calcium lines remain constant for all flares. This again supports the argument that these lines are emitted by a cool coronal plasma that cools in time in a similar manner for all flares (Sect. 3). However, the slight difference in the slope of the correlation to unity implies that the $\mathrm{H}_{\gamma}$ line to $\mathrm{Ca}$ II $\mathrm{K}$ line ratio varies from 1.95 to 3.39 over the entire energy range. This may be due to the fact that a radiative pumping process also takes place during the gradual phase for the large flares. Indeed, the plasma also emmits a substantial amount of radiation in the Balmer recombination continuum that will stimulate the Balmer line emission from the surrounding chromosphere. This will produce additional Balmer line emission during the gradual phase. 

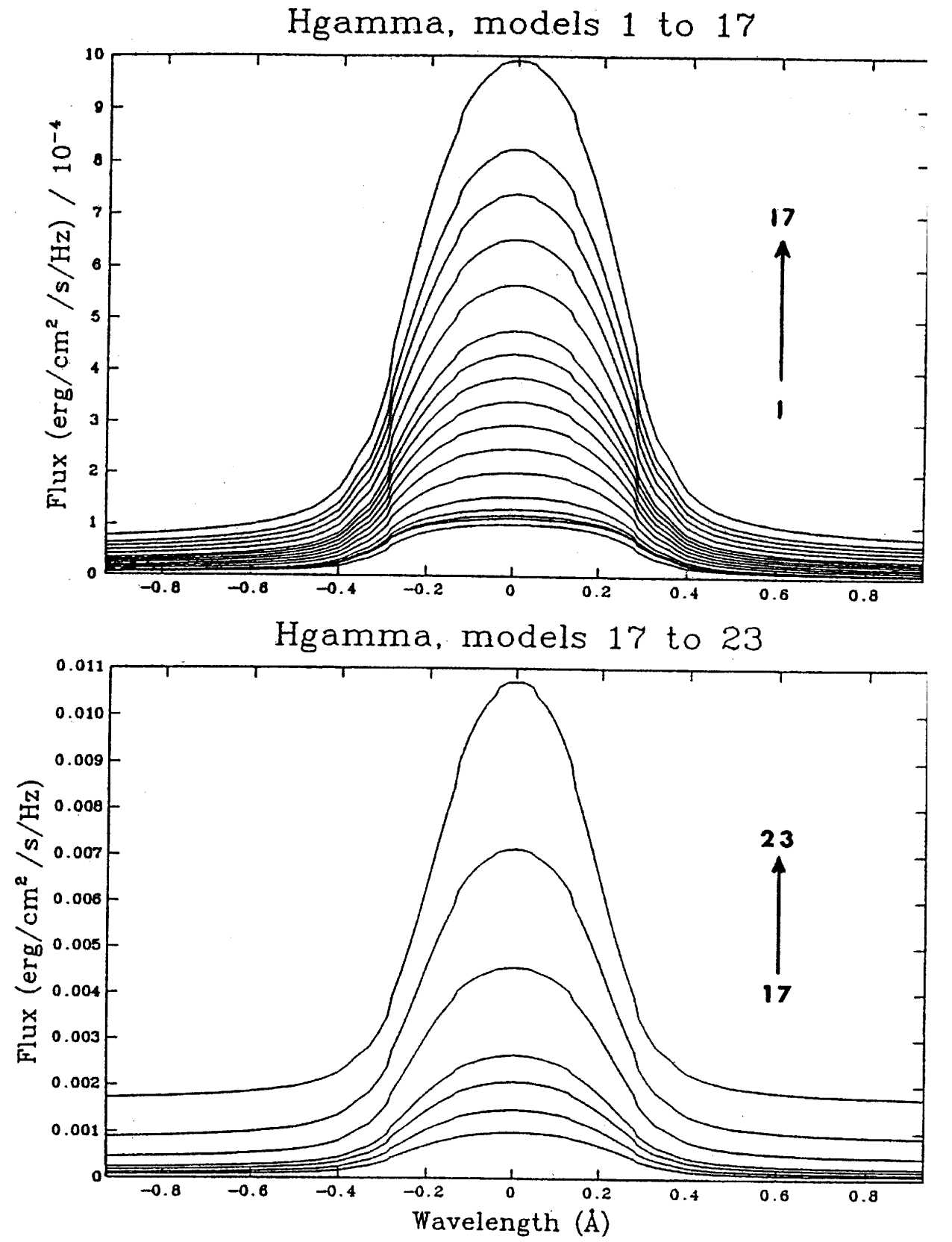

Fig. 4. This figure shows the variations in the $\mathrm{H}_{\gamma}$ line profiles when the atmosphere is illuminated by an external white light source at about the Balmer continuum (from H90). Model 1 is the quiescent atmosphere with no irradiation. Then the irradiation intensity increases from model 2 to 23 .

Figure 3e shows the $\mathrm{H}_{\gamma}$ line flux during the gradual phase as a function of the $\mathrm{H}_{\gamma}$ line flux during the impulsive phase. The slope of the correlation is 0.93 (Table 2), again very close to 1 . This is further evidence that the emission produced during the gradual phase directly depend on the impulsive phase. This also agrees with the finding that the Balmer line fluxes are correlated with the calcium line fluxes (Fig. 3d) and that both lines are correlated to the $U$-band (Figs. $3 \mathrm{~b}$ and 3c).

We also investigated the behaviour of the Balmer to Ca II flux line ratio (Table 4) as a function of the $U$-band energy and the time delays. We found no correlation between the line ratio and other parameters. The line ratio is usually between 2 and 4 except for a couple of flares (Table 4). This means, as highlighted by other correlations (e.g. Fig. 3d), that both the Balmer lines and Ca II lines grow simultaneously when the whole flare energy grows. The reason why, in a couple of flares, the Ca II emission is abnormally weak is unclear. Maybe in these instances the proportion of higher temperature plasma remains higher, and the flare plasmas do not undergo a normal cooling process, as discussed in Sect. 3.

\section{Maximum fluxes as a function of time lags}

In Fig. 6a we plot the rise time in the $\mathrm{Ca}$ II $\mathrm{K}$ line as a function of the $U$-band flux. As the correlation does not seem linear, we performed a fit with a second degree polynomial 
Table 4. Maximum fluxes during the impulsive and gradual phases of the flare for the $\mathrm{H}_{\gamma}$ line, Ca II $\mathrm{K}$ line and the $U$-band. We also give the ratios of the spectral line fluxes.

\begin{tabular}{lcccccccc}
\hline \hline Star & UT & Date & $\begin{array}{c}U \text {-band } \\
\text { max. flux } \\
(\mathrm{erg} / \mathrm{s})\end{array}$ & $\begin{array}{c}\mathrm{H}_{\gamma} \\
\text { max. flux } \\
\text { Imp. } \\
(\mathrm{erg} / \mathrm{s})\end{array}$ & $\begin{array}{c}\mathrm{H}_{\gamma} \\
\text { max. flux } \\
\text { Grad. } \\
(\mathrm{erg} / \mathrm{s})\end{array}$ & $\begin{array}{c}\text { Ca II K } \\
\text { max. flux } \\
(\mathrm{erg} / \mathrm{s})\end{array}$ & $\begin{array}{c}\text { Ratio } \\
\text { H I/Ca II } \\
\text { Imp./Grad. }\end{array}$ & $\begin{array}{c}\text { Ratio } \\
\text { H I imp./ } \\
\text { HI Grad. }\end{array}$ \\
\hline UV Ceti & $11: 19$ & $08 / 09 / 79$ & $2.9 \mathrm{E} 29$ & $2.1 \mathrm{E} 27$ & - & $1.6 \mathrm{E} 26$ & $13 /-$ & - \\
Prox Cen & $8: 21$ & $25 / 03 / 84$ & - & - & $1.4 \mathrm{E} 26$ & $4.5 \mathrm{E} 25$ & $-/ 3.1$ & - \\
Prox Cen & 8.50 & $25 / 03 / 84$ & $5.3 \mathrm{E} 26$ & $1.9 \mathrm{E} 26$ & - & $4.5 \mathrm{E} 25$ & $4.2 /-$ & - \\
AD Leo & $2: 40$ & $28 / 03 / 84$ & $1.7 \mathrm{E} 29$ & $1.6 \mathrm{E} 27$ & - & $<2 \mathrm{E} 26$ & $>8 /-$ & - \\
AD Leo & $3: 22$ & $28 / 03 / 84$ & $7.7 \mathrm{E} 29$ & $4.8 \mathrm{E} 27$ & $4.8 \mathrm{E} 27$ & $1.1 \mathrm{E} 27$ & $4.5 / 4.5$ & 1.0 \\
UV Ceti & $1: 53$ & $06 / 12 / 84$ & - & - & $9.1 \mathrm{E} 25$ & $6.8 \mathrm{E} 25$ & $-/ 1.3$ & - \\
UV Ceti & $2: 03$ & $06 / 12 / 84$ & - & - & $6.5 \mathrm{E} 25$ & $3.5 \mathrm{E} 25$ & $-/ 1.9$ & - \\
UV Ceti & $2: 29$ & $06 / 12 / 84$ & - & - & $1.5 \mathrm{E} 26$ & $6.1 \mathrm{E} 25$ & $-/ 2.5$ & - \\
UV Ceti & $2: 43$ & $06 / 12 / 84$ & $1.5 \mathrm{E} 27$ & $2.0 \mathrm{E} 26$ & - & $8.4 \mathrm{E} 25$ & $2.4 /-$ & - \\
UV Ceti & $3: 16$ & $06 / 12 / 84$ & - & - & $1.2 \mathrm{E} 26$ & $9.6 \mathrm{E} 25$ & $-/ 1.3$ & - \\
UV Ceti & $3: 29$ & $06 / 12 / 84$ & $2.2 \mathrm{E} 27$ & $2.6 \mathrm{E} 26$ & - & $7.7 \mathrm{E} 25$ & $3.4 /-$ & - \\
YZ CMi & $19: 55$ & $04 / 03 / 85$ & $4.4 \mathrm{E} 28$ & $9.0 \mathrm{E} 26$ & - & $5.7 \mathrm{E} 26$ & $1.6 /-$ & - \\
AD Leo & $4: 40$ & $12 / 04 / 85$ & $8.6 \mathrm{E} 30$ & $7.1 \mathrm{E} 28$ & $1.0 \mathrm{E} 29$ & $2.6 \mathrm{E} 28$ & $2.7 / 3.9$ & 0.71 \\
AT Mic & $1: 12$ & $20 / 08 / 85$ & $7.7 \mathrm{E} 30$ & $9.6 \mathrm{E} 28$ & $1.3 \mathrm{E} 29$ & $3.2 \mathrm{E} 28$ & $3.0 / 4.0$ & - \\
T48 & $13: 00$ & $04 / 12 / 85$ & - & - & - & - & - & - \\
II Peg & $2: 10$ & $17 / 08 / 89$ & $6.4 \mathrm{E} 31$ & $6.7 \mathrm{E} 29$ & $5.2 \mathrm{E} 29$ & $1.9 \mathrm{E} 29$ & $3.5 / 2.7$ & 1.3 \\
Gl 866 & $3: 27$ & $11 / 06 / 91$ & $6.5 \mathrm{E} 27$ & $1.6 \mathrm{E} 26$ & - & $7.0 \mathrm{E} 25$ & $2.3 /-$ & - \\
G1 866 & $3: 56$ & $11 / 06 / 91$ & $8.1 \mathrm{E} 27$ & $3.7 \mathrm{E} 26$ & - & $3.7 \mathrm{E} 25$ & $10 /-$ & - \\
G1 866 & $4: 24$ & $11 / 06 / 91$ & - & $4.3 \mathrm{E} 26$ & - & $9.2 \mathrm{E} 25$ & $4.7 /-$ & - \\
AD Leo & $0: 45$ & $14 / 03 / 98$ & $1.9 \mathrm{E} 30$ & $9.0 \mathrm{E} 28$ & $(3.5 \mathrm{E} 28)$ & $3.4 \mathrm{E} 28$ & $2.7 / 1.0$ & 2.6 \\
\hline
\end{tabular}

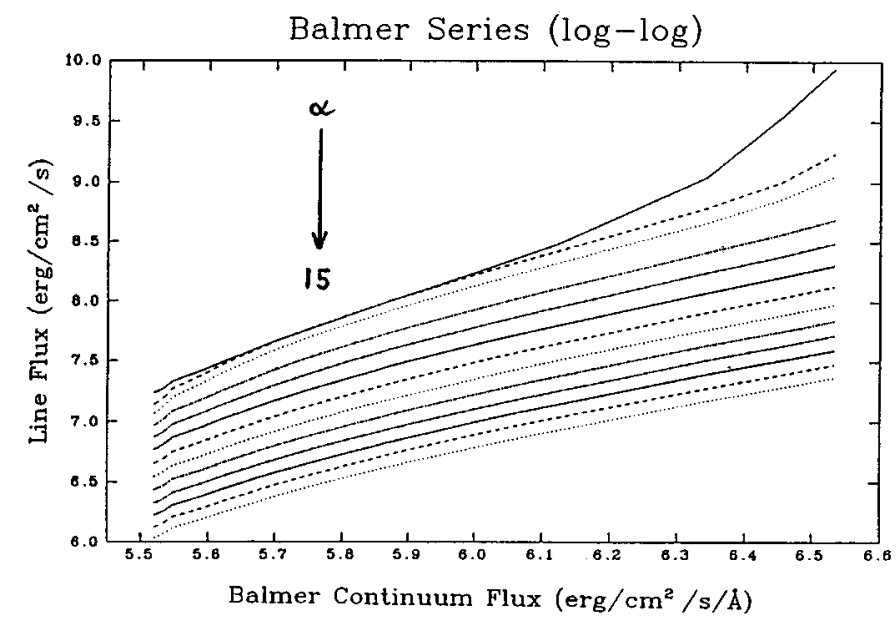

Fig. 5. We plot here the variations in the fluxes of the Balmer series as a function of the flux in the Balmer continuum at threshold, when an excess white light at about the Balmer continuum irradiates the quiescent atmosphere (from H90). These variations can be compared to the empirical correlation in Fig. 3a.

(Table 2). It shows that the more energetic the flare is, the longer the rise time in the gradual phase, or in other words, large flares last longer than small flares. This property is rather puzzling, it means, according to Gurzadyan (1984) models, that the plasma during the gradual phase has a lower density for large flares than for small flares. Figures $6 \mathrm{~b}$ and $4 \mathrm{c}$ show a similar trend although in these cases the correlations seem linear. This agrees with our finding that we did not have linear correlations for other relationships involving the $U$-band
(Figs. 3a and 3b) whereas we had linear correlations with line fluxes (e.g. Figs. 3c and 3d).

As mentioned above, the reason why larger flares should have lower densities is unclear. In the framework of flare plasma cooling models (Gurzadyan 1984) one possibility would be that the flare plasma has a higher initial temperature for larger flares. It would then take longer for the plasma to cool down and reach the maximum emisivity (Gurzadyan 1984), hence the rise times in the Balmer and calcium lines would be lengthened. But if the density was the same for small and large flares then the decay times should be identical, which is not observed (Figs. $2 \mathrm{~b}$ to $2 \mathrm{~d}$ and $6 \mathrm{~d}$ ). There is therefore no simple explanation with the present models as to why the larger flares last longer and why they have lower densities during the gradual phase. An alternative explanation is that secondary energy release takes place during large flares which lengthens their duration.

It is also interesting to note that the larger flares on RS CVn systems, even though they may have somewhat different properties, last even longer (e.g. Baliunas et al. 1984; Linsky et al. 1989; Foing et al. 1994). The correlations found here (Figs. 6a to 6c) may therefore extend to flares on RS CVn systems.

\section{Balmer line broadening versus white light}

Spectral lines of different species exhibit quite different behaviour in their broadening during a flare (H92a, Hawley $\&$ Pettersen 1991; Garcia-Alvarez 2001). This aspect of the flare as well as possible broadening mechanisms were already extensively commented by H92a. Here, we will focus our 

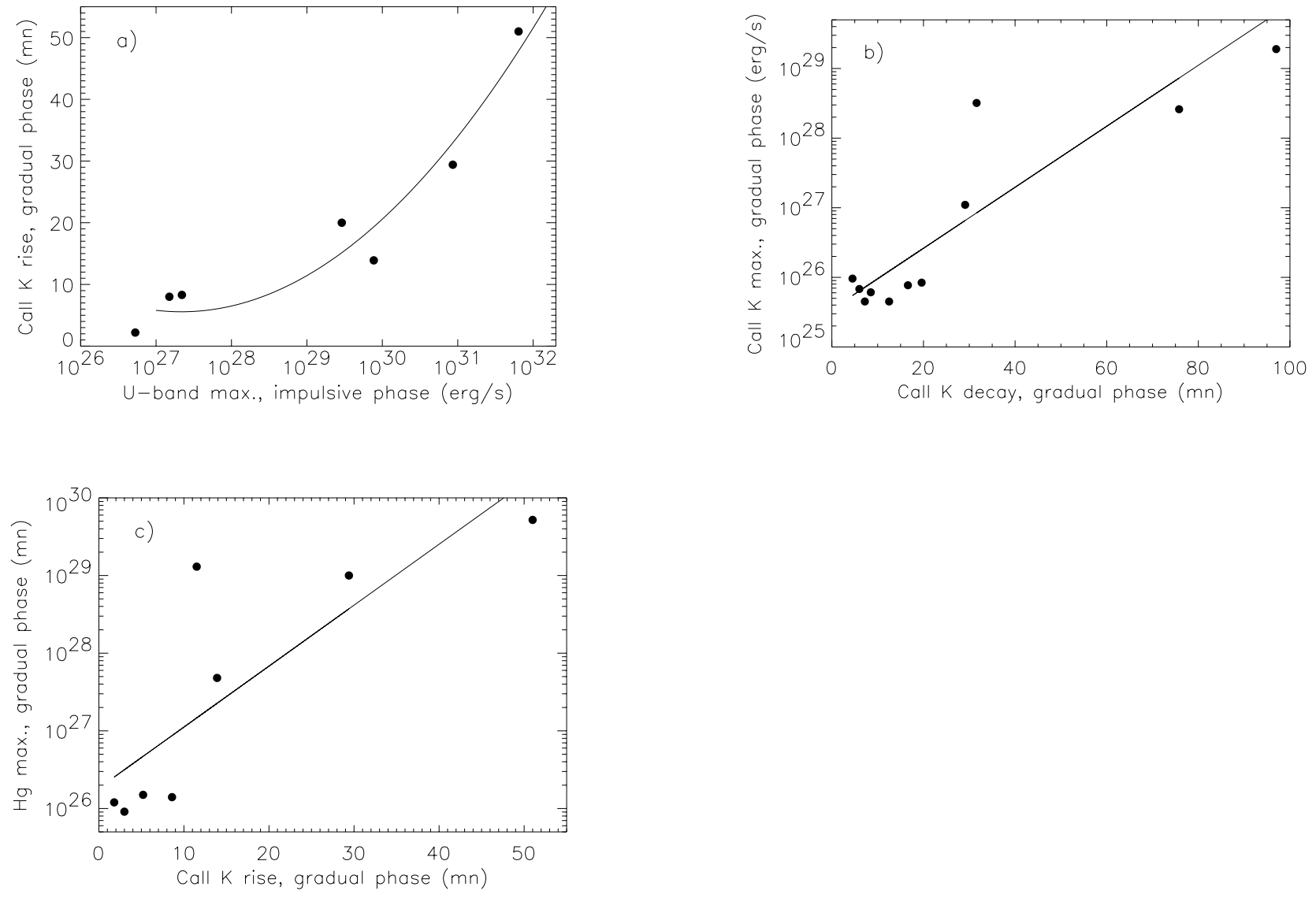

Fig. 6. In figures a) to c), we show the $U$-band and line maximum fluxes as a function of delays in the spectral lines. The two parameters correlate which shows that basically, large flares last longer than small flares.

attention on how the broadening in the Balmer lines varies with the $U$-band flux. Previous evidence for Stark and opacity broadening suggest the presence of high density kernels during the flare impulsive phases (H92a). Has the density of these kernels any dependence on the magnitude of the flare? Large broadening seems to coincide with powerful white light emission, with the maximum attained during maximum white light (H92a, Hawley \& Pettersen 1991; Garcia-Alvarez 2001). This maximum is followed by a monotonic decay of the line widths. There seems therefore to be some degree of correlation between the line width and the white light intensity during a flare.

H92a already investigated the dependence of the Balmer line widths on the white light intensity. Here we augment his dataset with other observations. We show in Fig. 7 the FWHM of $\mathrm{H}_{\gamma}, \mathrm{H}_{\delta}, H_{8}$ and $H_{9}$ versus the $U$-band flux for flares on AD Leo (2:40 and 3:22 UT on 28 March 1984, Rodonò et al. 1989, 4:40 UT on the 12 April 1985, Hawley \& Pettersen 1991), YZ CMi (Doyle et al. 1988a; Zarro \& Zirin 1985), UV Ceti (de Jager et al. 1989; Phillips et al. 1988), Proxima Cen (PC2 flare) and AT Mic (Garcia-Alvarez et al. 2001). Filled circles are for the impulsive phase only, open circles are for the gradual phase.

Broadening during the impulsive phase is much larger in average than during the gradual phase. Typical values are respectively in the range [5 $⿻ 16 \AA]$ and $[2 \AA ; 7 \AA]$. Note that the lower limit of $2 \AA$ is often set by the instrumental resolution. The data shows a clear trend for increased broadening with white light intensity, both during the impulsive and gradual phases. In fact, we may distinguish three energy domains for the impulsive phase measurements in this diagram: the small flares with $U$-band energies lower than $\sim 10^{25} \mathrm{erg} / \mathrm{A}$, the medium size flares from $\sim 10^{25} \mathrm{erg} / \AA$ to $\sim 3 \times 10^{26} \mathrm{erg} / \AA$, and the large flares for energies larger than $\sim 3 \times 10^{26} \mathrm{erg} / \AA$. For small flares, we believe that the kernels may not reach a sufficiently high density so as to dominate the Balmer line emission. In this case, the Balmer emission originates from various areas such as in solar two-ribbon flares, and the broadening is of the same order than that observed during the gradual phase of larger flares. In other words, as in Sect. 4 we propose that observations are evidence that the $U$-band is no longer a good tracer of the energy released during the flare. For $U$-band energies above $\sim 10^{25} \mathrm{erg} / \AA$, the $F W H M$ clearly increases as a function of energy up to a value of about $15 \AA$. This increase is in agreement with the results obtained for the kernel model atmospheres (H92a), and shows that, the larger the flare, the larger is the pressure in the flare kernels, and therefore the higher is the energy of the particle beams.

For large flares ( $U$-band energies above $\sim 3 \times 10^{26} \mathrm{erg} / \AA$ ) , the situation seems more puzzling. As was shown in Sect. 4.2, we can interpret the data for large flares in Fig. 3a only if we invoke an increasing contribution to the Balmer lines arising 


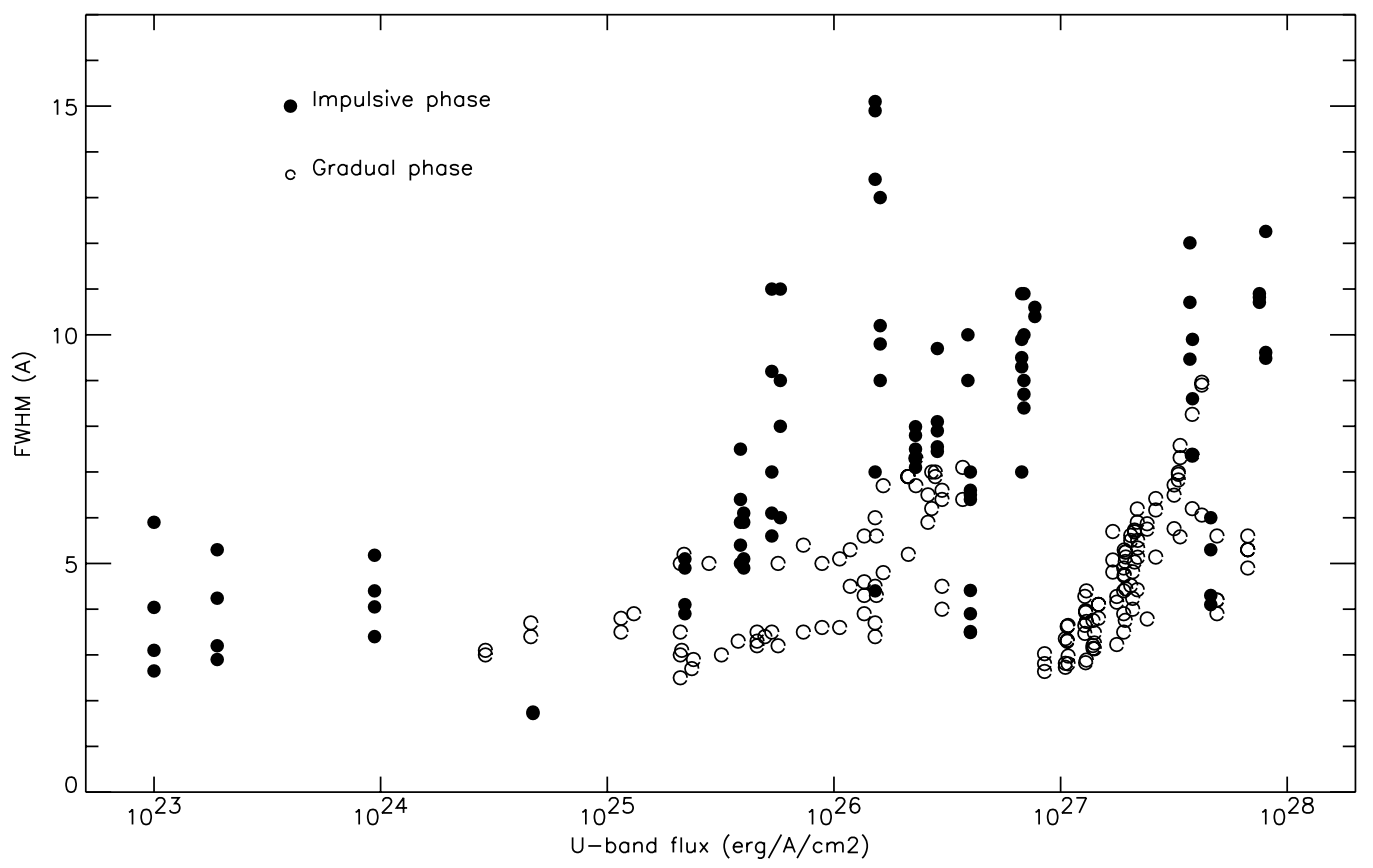

Fig. 7. $F W H M$ of the $\mathrm{H}_{\gamma}, \mathrm{H}_{\delta}, H_{8}$ and $H_{9}$ lines versus $U$-band flux. We included all the data available. Measurements corresponding to the impulsive phase and gradual phase are reported respectively as filled and open circles. An obvious trend is depicted in the $2 \times 10^{25}-3 \times$ $10^{26} \mathrm{erg} / \AA \mathrm{A}$ range that we believe is evidence for increasing density in the flare kernels, and therefore increasing energy and intensity of particle beams.

from the radiative pumping effect on the surrounding chromosphere. If this indeed occurs, then we should observe a progressive decrease in the Balmer maximum widths as the $U$-band energy increases because the Balmer lines are not broadened by this process. This is indeed what we observe in Fig. 7 for the flare on AD Leonis (Hawley \& Pettersen 1991). However for the flare on AT Mic (Garcia-Alvarez et al. 2001) we observe a broadening that is still quite large; about $10 \AA$. Therefore, for $U$-band energies above $\sim 3 \times 10^{26} \mathrm{erg} / \AA$ it seems that there is an increasing contribution from the radiative pumping process for some flares but not all.

A large scattering in $F W H M$ for a given $U$-band energy is not only expected because of a probable large variety in the flare phenomenon, but also because of the changing sites for flare occurrence (disk and limb flares); indeed, according to the model calculations, the modification of the flare integrated intensities and line profiles is large for different propagation angles when the plasma density is high (H92a).

It is reasonable to assume in first approximation that the maximum intensity of suspected particule beams scale with the strength of the magnetic field in the flaring loops. Now, magnetic loops on a dMe star are limited in strength, to maybe a few KG or tens of KG. This saturation in the field strength will cause the particle beams to saturate in energy for a given amount of energy released, or for a given $U$-band flare intensity (assuming the $U$-band is a good tracer of the total energy budget). In addition, because the chromospheric pressure depends primarily on the total energy deposited, then the maximum chromospheric pressure will also be limited for a given $U$ band energy. On account of the dependence of the line widths with chromospheric pressure (H92a), thus, we expect such a scatter diagram to delineate an upper envelope in the FWHM for a given white light flux. This envelope is visible in Fig. 7, and the line $F W H M$ clearly increase with the $U$-band flux. Assuming that the kernel contribution dominate the Balmer line flux, this maximum envelope defines the maximum particle beam intensity for a given U-band flux. It is therefore possible in principle to recover from Fig. 7 the maximum beam energy and intensity as a function of the $U$-band flux.

It is important to note that the rise in the line widths is very rapid in the $\sim 10^{25} \mathrm{erg} / \AA$ to $\sim 3 \times 10^{26} \mathrm{erg} / \AA$ energy range. What happens at energies higher than $\sim 3 \times 10^{26} \mathrm{erg} / \AA$ ? First of all lets investigate how the Balmer contribution from the flare kernels can evolve towards higher energies. Lets investigate the two cases, when the magnetic field strength saturates and when it does not, and therefore when the particle beam energy saturates and when it does not. If it indeed saturates, then, so will the maximum chromospheric pressure and the line width. In this case larger flares are due to larger flaring areas and not to a higher beam intensity or energy. If the kernels are still the main contributor to the Balmer emmission, then we should observe a plateau (saturation) in the line widths at higher energies. The situation is not clear because of a lack of observations, but according to Fig. 7 there may be a saturation in the widths, if not a decrease for large flares. This indicates that larger flares involve larger areas rather than higher energy particle beams. On the other hand, the AD Leo flare observed by Hawley \& Pettersen (1991) indicates that for some flares, as already found for the Balmer flux (Sect. 4.2 and Fig. 3a), the contribution to the Balmer emission due to other sources (radiative pumping, backwarming, lower density coronal plasmas) increasingly dominate. 
If the particle beam energy does not saturate, then the maximum chromospheric pressure will increase with increasing $U$ band flux: the very rapid rise in the line widths implies that a width of $\sim 37 \AA$ will be reached for a $U$-band flux of $10^{28} \mathrm{erg} / \AA$ ! Indeed, for chromospheric pressures even higher than those studied by H92a, Stark broadening dominates the line formation and yields very broad profiles. Moreover, opacities become also very large and the line formation approaches that of a black-body. In other words, the Balmer lines progressively vanish in strength (because of opacity) and become very broad because of the large Stark broadening. Therefore, we are in a situation where the line equivalent widths fall with increasing $U$-band intensity and become so broad that they would appear to contribute only to the line wings. Hence, in the case when the beam energy does not saturate, the contribution from the kernels, naturally vanishes out of sight such that for large flares there remains only the contributions from other flare plasmas, except in the line wings. Here, we have a natural explanation so as why in some flares the observed line widths fall with energy for energies larger than $\sim 3 \times 10^{26} \mathrm{erg} / \AA$.

To conclude, we find that; (i) for energies lower than $10^{25} \mathrm{erg} / \AA$, flare kernels are not dense enough to dominate the Balmer emission and the $U$-band is not a good tracer of the flare energy, (ii) for energies in the range $\sim 10^{25} \mathrm{erg} / \AA$ to $\sim 3 \times 10^{26} \mathrm{erg} / \mathrm{A}$, the flare kernels dominate the Balmer emission and give rise to a rapid increase in the line widths, (iii) for energies larger than $\sim 3 \times 10^{26} \mathrm{erg} / \AA$, the situation is not clear. The kernel contribution may progressively vanish, either naturally because the chromospheric pressure still increases, or because the contribution of other plasmas increases, or both. But it seems that for some flares the kernel contribution is still large, and such flares may be scaled up versions of medium size flares. In this case we should observe a saturation in the line widths.

It is important to remark that in any case the widths attain a maximum for $U$-band energies of the order of $\sim 3 \times 10^{26} \mathrm{erg} / \AA$, and do not increase for higher energies. This shows that either large flares are scaled up versions of medium size flares, or that the contribution to the Balmer emission due to other sources than the kernels (radiative pumping, backwarming, lower density coronal plasmas) dominate.

We may also note that the gradient depicted for gradual phase measurements also suggests that densities or turbulence in non-kernel plasmas increase with the flare magnitude. This could indicate that backwarming effects on the atmosphere surrounding the flare are also magnified. Here again, the large scatter points to the probability that varying processes are at work.

\section{The Balmer line fluxes as a function of white light flux}

We compiled all the simultaneous data of the Balmer lines and the $U$-band, during the impulsive phase. The results are shown in Fig. 8. In this figure we included the flares on; AD Leonis, 28/03/1984 2:40 UT and 3:22 UT, 12/04/1985 4:40 UT, 14/03/1998 0:45 UT, UV Ceti 08/09/1979 11:19 UT, 06/12/1984 2:43 UT and 3:29 UT, Prox Cen 25/03/1984 8:50 UT, YZ CMi 04/03/1985 19:55 UT, Gl 866 11/06/1991
3:27 UT and 3:56 UT, AT Mic 20/08/1985 1:12 UT. This diagrame is similar to Fig. 3a except that here we included all the points during the impulsive phase and not only the maximum flux.

When only the continuum flux measurement was available in spectrophotometry, we transformed the continuum flux into $U$-band flux assuming (Lacy et al. 1976):

$E_{U-\text { band }} \simeq 1.20 \pm 0.08 E_{B-\text { band }}$

where $E_{U \text {-band }}$ and $E_{B \text {-band }}$ are respectively the flare $U$-band energy and the flare $B$-band energy.

Observations of the impulsive phase are correlated, and we fitted this correlation with a linear relation (Table 2). On the contrary, observations of the gradual phase are not correlated, and in fact the impulsive phase delineates a lower limit in the Balmer line fluxes for a given $U$-band flux. It is interesting to note that this corrrelation is valid for flares that span nearly 5 orders of magnitude in the $U$-band and that occur on different stars. This emphasizes that a universal mechanism is at work during the impulsive phase, that relates the formation of the $U$-band and the Balmer lines from the weakest flares to the largest ones. Other similar correlations between soft X-rays and radio fluxes or soft X-rays and Balmer line fluxes (Butler 1993; Benz \& Gudel 1994) also indicate that emissions at different wavelengths are intimately related.

H92a showed that two leading mechanims could be responsible for the observed correlation; i) the dense flare kernels, and ii) the radiative pumping of the surrounding chromosphere by the white light. In the case of the flare kernels, both the white light intensity and the Balmer line intensity grow when the electron pressure increases, and because their growth is directly dependent on electron pressure, the two are correlated. However, H92a models cover only one order of magnitude in the $U$-band flux and cannot therefore explain the entire correlation. Hence, there is a need for a larger grid of model atmospheres, especially towards lower chromospheric pressures. It is important to note that, although larger flares are expected to cover larger areas, a simple scaling of the smaller flares cannot explain the observations. Indeed, if large flares were simply scaled up versions of smaller flares, then the correlation would have a slope of one. Even though the slope tends to increase towards the end of the energy range (Fig. 3a), the slope is generally smaller over most of the observed domain. Moreover we obtained a slope of 0.62 for the observations reported in Fig. 8 (Table 2). Therefore, simple scaling cannot explain the observations.

Models that mimic the effect of the radiative pumping by the white light on the chromosphere produce correlations with a slope between 0.6 and 1.7 (Houdebine 1992a; Houdebine 1990). Moreover they can cover more than three orders of magnitude in the $U$-band intensity. The M.A.1 series of model atmospheres in $\mathrm{H} 90$ agree rather well with observations, giving a slope close to the one observed in most of the $U$-band energy range. However, according to the variation of the Balmer FWHM as a function of $U$-band (Fig. 7) it is unlikely that this process is entirely responsible for the observed correlation between the Balmer line flux and the $U$-band flux. We propose that there is a combination of the two mechanisms as well as 


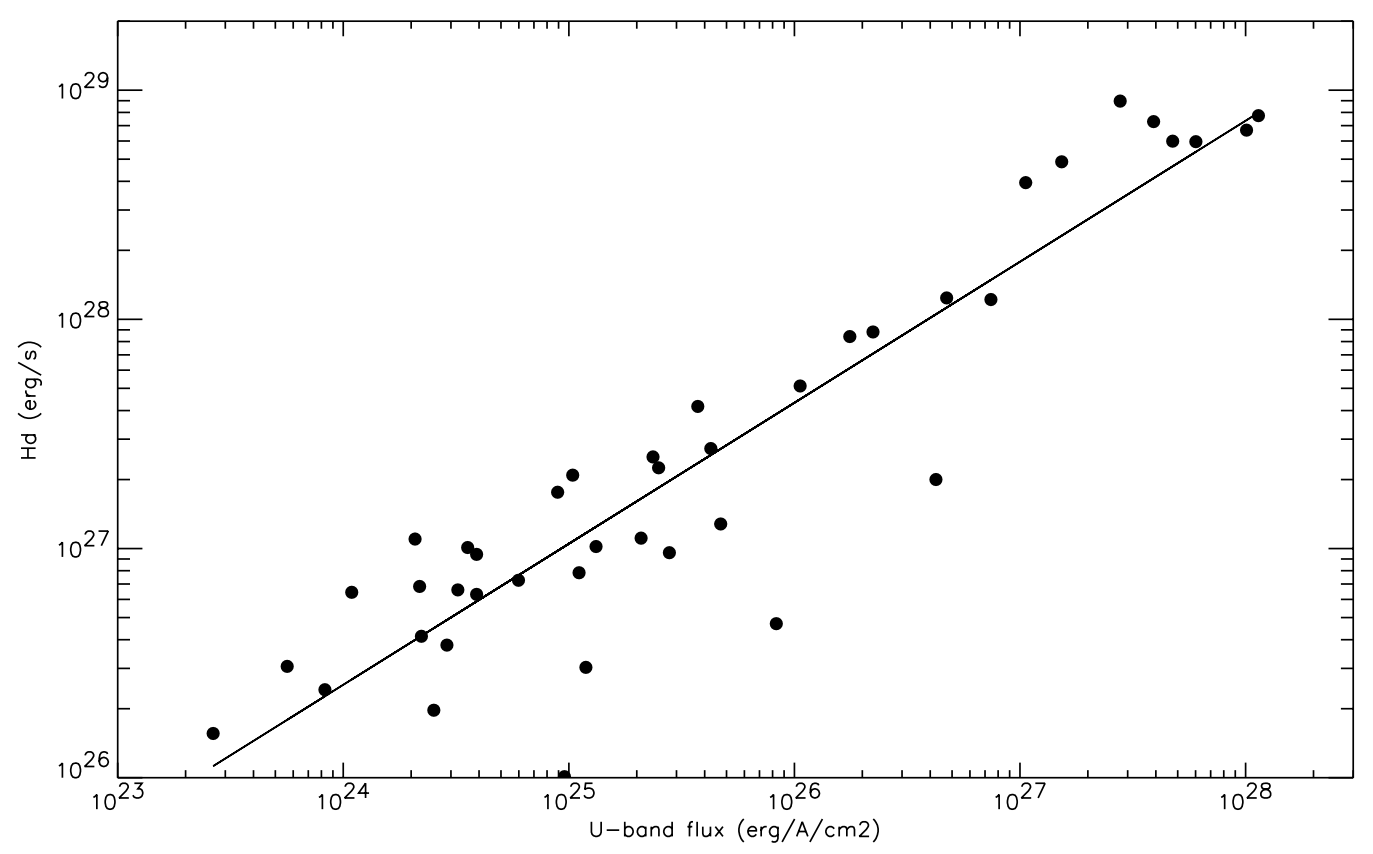

Fig. 8. Flux in the $\mathrm{H}_{\delta}$ line as a function of the $U$-band flux during the impulsive phase of flares. The data correlate over 3 orders of magnitude, but they also correlate during the impulsive phase of each flare, thus showing that the Balmer and continuum emissions are intimately related.

an increasing area of the flare at the lower atmospheric levels (kernels).

\section{Conclusion}

We compiled all the available data sets that include timeresolved spectroscopy in the $3600 \AA-4500 \AA$ wavelength range. We first analysed the flare time profile characteristics in terms of impulsive and gradual phases, and found a general description that holds for all the flares observed: namely, the flare is composed of an impulsive phase and a gradual phase which have different physical properties, and which arise in different proportions for different flares. The main signature of the impulsive phase is an impulsive behaviour in the Balmer line fluxes, always accompanied by a white light counterpart. The gradual phase evolves more slowly and has no white light counterpart. We found that all large flares are combined flares, i.e., they have strong distinct impulsive and gradual phases. Conversely, small flares can appear either totally dominated by the gradual or the impulsive phase.

We measured the rise and decay times in the time profiles of the Balmer and Ca II lines. We showed that the time profiles obey very precise laws that are valid for all flares in spite of their great variety in spectral signatures. These laws provide important constraints for modelling the flare phenomenon, future models will have to predict spectral line time-profiles that are identical for flares that have decay times from a couple of minutes to a couple of hours or more. In this respect, we found that the simple thermal cooling model proposed by Gurzadyan (1984, see also Houdebine et al. 1991) can reproduce very simply the observed features. However, in this case, observations also imply that small flares are compact and dense while larger flares have a lower density, which remains rather puzzling. In addition, we found that the constant ratio in the delays of the line time-profiles seem difficult to explain with X-ray backwarming models of the chromosphere.

We measured the maximum fluxes in the $U$-band and in spectral lines for both the impulsive and the gradual phases. The $U$-band and Balmer line fluxes correlate very well over more than 5 orders of magnitude. Moreover, the correlation is not linear and the gradient is generally smaller than 1 , which shows that large flares are not simply scaled up versions of smaller flares, but that precise mechanisms that control both the $U$-band and the Balmer line formation are at work. As far as these mechanims are concerned, we establish that the flare kernel models and the radiative pumping models of Houdebine (1990, 1992a) can be combined to reproduce the observations. Other correlations between the spectral line fluxes, and the $U$ band flux, essentially demonstrate that the fluxes during the gradual phase and the impulsive phase grow together by the same rate.

Further correlations between the fluxes and the time delays in the spectral lines are evidence that, the more powerful the flare, the longer it lasts. As mentioned above, in the framework of Gurzadyan (1984) models, this implies that, the more powerfull the flare, the lower its density, at least for the plasmas concerned during the gradual phase. This remains unexplained at this stage. An alternative explanation is that more secondary energy release take place during the gradual phase of large flares, than during smaller flares.

The study of the Balmer line full width half maximum shows that the picture is likely to be more complex than expected. It seems that the description of the flares is best when divided into three subgroups; the small flares, the medium size flares, and the large flares. For the former, line broadening remains small and does not seem to depend on the flare $U$-band energy, probably because this latter feature is not a good diagnostic of the whole energy released. 
For intermediate energy flares, the line widths increase rapidly with the $U$-band energy, which seems evidence that, at this stage, emission from dense kernels dominates the overall Balmer emission. For larger flares, the line widths seem to reach a plateau. We interpret this as being a consequence of the kernel Balmer signature becoming very broad and vanishing away, and, as the radiative pumping process becomes more efficient, progressively taking over the Balmer emission, or because larger flares are simply scaled up versions of medium size flares.

Finally, we investigated in more detail the Balmer emission during the impulsive phase as a function of the $U$-band emission. We find that the correlation between the two parameters during the impulsive phase does in fact delimit a lower boundary in the Balmer- $U$-band diagram. The data shows that the Balmer emission and the $U$-band emission during the impulsive phase are correlated. We understand this correlation as being the direct signature of both dense kernel emission and the radiative pumping process taking place in the surrounding chromosphere.

We found that the simple characteristics of the flare flux time-profiles in visible spectroscopy provide valuable constraints for the modelling of stellar flares. Moreover, our correlations provide new insights into solar flare physics, and we suggest that observations of the sun as a star (whole sun observations) should be performed in the future to allow further comparison between solar and stellar flares. Future spectroscopic observations of flares, should be performed with a sufficiently high time resolution (at least $10 \mathrm{~s}$ ), in particular if one wants to investigate the impulsive phase in detail. It would also be of particular interest to study the behaviour of other spectral lines, especially higher temperature transition region lines (such as the helium lines) and lower temperature lines such as $\mathrm{Mg}$ II or $\mathrm{Ca}$ I lines, in order to find out if similar correlations apply to them. Finally, future models will have to account for the numerous correlations obtained in this study, that apply to all flares.

Acknowledgements. This work was supported by a PPARC Research Fellowship (Grant PPA/G/S/2000/00076).

\section{References}

Allard, F., \& Hauschildt, P. H. 1995, ApJ, 445, 433

Allen, C. W. 1973, Astrophysical Quantities (Athlone Press - London) Baliunas, S. L., Guinan, E. F., \& Dupree, A. K. 1984, ApJ, 282, 733

Benz, A. O., \& Gudel, M. 1994, A\&A, 285, 621

Bruevich, E. A., Katsova, M. M., \& Livshits, M. A. 1990, Sov. Astron., 34,614

Butler, C. J. 1993, A\&A, 272, 507

Butler, C. J., Rodonò, M., Foing, B. H., \& Haisch, B. M. 1986, Nature, 321,679

Butler, C. J., Doyle, J. G., Foing, B. H., \& Rodonò, M. 1988, Activity in Cool Star Envelopes, ed. O. Haines, B. Pettersen, J. Schmitt, \& J. Solheim, 167

Butler, C. J., et al. 2002, in preparation

Carter, B. D., O'Mara, B. J., \& Ross, J. E. 1988, MNRAS, 231, 49

de Jager, C., Heise, J., van Genderen, A. M., et al. 1989, A\&A, 211, 157
Doyle, J. G., Butler, C. J., Byrne, P. B., \& van den Oord, G. H. J. 1988b, A\&A, 193, 229

Doyle, J. G., Kellet, B. J., Butler, C. J., et al. 1992, A\&AS, 96, 351

Eason, E. L. E., Giampapa, M. S., Radick, R. R., Worden, S. P., \& Hege, E. K. 1992, AJ, 104, 1161

Foing, B. H., Char, S., Ayres, T., et al. 1994, A\&A, 292, 543

Garcia-Alvarez, D., Jevremovic, D., Doyle, J. G., \& Butler, C. J. 2001, A\&A, submitted

Gliese, W. 1969, Catalogue of Nearby Stars, Veroff. Astron. Recher-Institut Heidelberg, No. 22

Gliese, W. 1991, augmented Gliese Catalogue from the IDL Library

Gunn, A. G., Doyle, G. J., Mathioudakis, M., Houdebine, E. R., \& Avgoloupis, S. 1994, A\&A, 285, 489

Gurzadyan, G. A. 1984, Ap\&SS, 106, 1

Hawley, S. L., \& Pettersen, B. R. 1991, ApJ, 378, 725

Hawley, S. L., Fisher, G. H. 1992, ApJS, 78, 565

Houdebine, E. R. 1990, Ph.D. dissertation, Université d'Orsay-Paris XI

Houdebine, E. R. 1992a, Irish Astron. J., 20, 213

Houdebine, E. R. 1992b, Cool Stars, Stellar Systems and the Sun, ed. M. S. Giampapa, \& J. A. Bookbinder, PASPCS, 26, 537

Houdebine, E. R., \& Doyle, J. G. 1994a, A\&A, 289, 169

Houdebine, E. R., \& Doyle, J. G. 1994b, A\&A, 289, 185

Houdebine, E. R., \& Stempels, S. 1997, A\&A, 326, 1143

Houdebine, E. R., Butler, C. J., Panagi, P. M., Rodonó, M., \& Foing, B. H. 1991, A\&A, 87, 33

Houdebine, E. R., Foing, B. H., Doyle, J. G., \& Rodonò, M. 1993a, A\&A, 259, 302

Houdebine, E. R., Foing, B. H., Doyle, J. G., \& Rodonò, M. 1993b, A\&A, 278, 109

Jevremovic, D. 1999, Ph.D. Thesis Dissertation, Queen's University Belfast

Jevremovic, D., Butler, C. J., Drake, S. A., O’Donoghue, D., \& van Wyk, F. 1998, A\&A, 338, 1057

Johnson, H. L. 1966, ARA\&A, 4, 193

Jones, H. R. A., Longmore, A. J., Jameson, R. F., \& Mountain, C. M. 1994, MNRAS, 267, 413

Katsova, M. M. 1990, Sov. Astron., 34, 614

Katsova, M. M., Livshits, M. A., Butler, C. J., \& Doyle, J. G. 1991, MNRAS, 250, 402

Katsova, M. M., Drake, J. J., \& Livshits, M. A. 1999, ApJ, 510, 986

Lacy, C. H., Moffet, T. J., \& Evans, D. S. 1976, ApJS, 30, 85

Legget, S. K. 1992, ApJS, 82, 351

Linsky, J. L., Neff, J. E., Brown, A., et al. 1989, A\&A, 211, 173

Mirzoyan, A. V. 1993, Astrofizica, 36, 277

Mould, J. R. 1976, A\&A, 48, 443

Phillips, K. J. H., Bromage, G. E., Dufton, P. L., Keenan, F. P., \& Kingston, A. E. 1988, MNRAS, 235, 573

Rodonó, M., Houdebine, E. R., Catalano, S., et al. 1989, Solar and Stellar Flares, Poster Paper Proc., special publication of Catania Astrophys. Obs., ed. B. M. Haisch, \& M. Rodonó, 53

Simon, T., Linsky, J., \& Schiffer, F. H. 1980, ApJ, 239, 911

Strassmeier, K. G., Hall, D. S., Fekel, F. C., \& Scheck, M. 1993, A\&AS, 100, 173

Tandberg-Hanssen, E., \& Emslie, A. G. 1988, The Physics of Solar Flares (Cambridge University Press)

Turon, C., and the INCA Consortium 1997, The Hipparcos and Tycho Catalogues, ESA SP-1200

Veeder, G. J. 1974, AJ, 79, 1056

Zarro, D. M., \& Zirin, H. 1985, A\&A, 148, 240 